ARTICLE

Received 16 Apr 2014 | Accepted 25 Sep 2014 | Published 31 Oct $2014 \quad$ DOl: 10.1038/ncomms6379

\title{
Functional characterization of the TERRA transcriptome at damaged telomeres
}

\author{
Antonio Porro ${ }^{1,2, \dagger}$, Sascha Feuerhahn ${ }^{1,2, \star, \dagger}$, Julien Delafontaine ${ }^{1,3, \star}$, Harold Riethman ${ }^{4}$, \\ Jacques Rougemont ${ }^{1,3} \&$ Joachim Lingner ${ }^{1,2}$
}

Telomere deprotection occurs during tumorigenesis and aging upon telomere shortening or loss of the telomeric shelterin component TRF2. Deprotected telomeres undergo changes in chromatin structure and elicit a DNA damage response (DDR) that leads to cellular senescence. The telomeric long noncoding RNA TERRA has been implicated in modulating the structure and processing of deprotected telomeres. Here, we characterize the human TERRA transcriptome at normal and TRF2-depleted telomeres and demonstrate that TERRA upregulation is occurring upon depletion of TRF2 at all transcribed telomeres. TRF2 represses TERRA transcription through its homodimerization domain, which was previously shown to induce chromatin compaction and to prevent the early steps of DDR activation. We show that TERRA associates with SUV39H1 H3K9 histone methyltransferase, which promotes accumulation of H3K9me3 at damaged telomeres and end-to-end fusions. Altogether our data elucidate the TERRA landscape and defines critical roles for this RNA in the telomeric DNA damage response.

\footnotetext{
${ }^{1}$ Ecole Polytechnique Fédérale de Lausanne (EPFL), School of Life Sciences, 1015 Lausanne, Switzerland. ${ }^{2}$ Swiss Institute for Experimental Cancer Research (ISREC) at EPFL, Station 19, CH-1015 Lausanne, Switzerland. ${ }^{3}$ Bioinformatics and Biostatistics Core Facility, EPFL and Swiss Institute of Bioinformatics, Station 15, CH-1015 Lausanne, Switzerland. ${ }^{4}$ The Wistar Institute, 3601 Spruce Street, Philadelphia, Pennsylvania 19104, USA. * These authors contributed equally to this work. †Present address: Institute of Molecular Cancer Research, Winterthurerstrasse 190, University of Zürich, Switzerland, CH-8057 Zürich, Switzerland. Correspondence and requests for materials should be addressed to J.R. (email: jacques.rougemont@epfl.ch) or to J.L. (email: joachim.lingner@epfl.ch).
} 
T he prevalent damage of the genetic material poses a particular threat to genome integrity and cell survival. Sensitivity of DNA to damaging agents and the kinetics of repair are influenced by the chromatin structure. The DNA damage response (DDR) differs in heterochromatic and euchromatic genomic loci. Heterochromatin is better protected from damage than euchromatin ${ }^{1}$ but damaged heterochromatin is less accessible to DDR factors. Thus, DNA damage repair in heterochromatin occurs with slow kinetics and is less effective than in euchromatin, resulting in long-term retention of DNA lesions ${ }^{2}$. While the ataxia-telangiectasia mutated protein (ATM) prevents large-scale chromatin decompaction and silences transcription in cis next to DNA double strand breaks (DSBs) in euchromatic domains ${ }^{3}$, repair of damaged heterochromatic regions is likely to require decondensation of the surrounding chromatin structure and derepression of transcription ${ }^{4,5}$. Chromatin relaxation and subsequent repair of heterochromatin requires the ATM-dependent phosphorylation of the heterochromatin protein Kap-1 (ref. 6) and the rapid release of phosphorylated HP1 proteins from H3K9me3 (ref. 7), followed by association of Tip60, an acetyltransferase involved in chromatin acetylation 8,9 and in the modification and subsequent activation of $\mathrm{ATM}^{10-12}$.

Telomeres resemble constitutive heterochromatin as they contain H3K9me3 and HP1 proteins ${ }^{13}$. Telomeres also actively repress DNA repair pathways to prevent recombination of natural chromosome ends. Consistent with these features, telomeres when damaged but still functional elicit a persistent $\mathrm{DDR}^{14}$ and they are refractory to DNA repair ${ }^{15}$, thus causing cellular senescence ${ }^{15}$. In vertebrates, telomeric DNA is associated with specialized telomeric proteins, known as shelterins ${ }^{16}$, which repress DNA damage activation at the ends of linear chromosomes ${ }^{17}$. At functional telomeres, the shelterin component TRF2 binds double-stranded telomeric DNA and represses ATM signalling, whereas the single-stranded telomeric binding factor POT1 prevents ATR (ataxia telangiectasia and Rad3 related) activation ${ }^{18}$. Extensive telomere shortening leads to activation of the ATM and ATR pathways and replicative senescence which is thought to suppress tumorigenesis ${ }^{19-22}$.

Telomeres in most or all eukaryotes are transcribed into heterogeneous long non-coding RNAs known as TERRA ${ }^{23,24}$. TERRA transcription is driven by RNA polymerase II (RNAPII) and proceeds from subtelomeric DNA into the telomeric repeat sequences ${ }^{23,24}$. In humans, $\mathrm{CpG}$ island promoters present at a subset of telomeres are partially responsible for driving TERRA transcription and PCR with reverse transcription (RT-PCR) analyses as well as FISH experiments suggested that several if not all telomeres can produce TERRA transcripts ${ }^{25}$. However, a comprehensive description of the whole TERRA transcriptome is missing. TERRA transcription has been linked to chromatin structure and is repressed by the DNA methyltransferase enzymes DNMT1 and DNMT3b 25,26 and by the SUV39H1 H3K9 histone methyltransferase and heterochromatin protein $1 \alpha$ (HP1 $\alpha$; ref. 27). TERRA partially colocalizes with telomeres ${ }^{23,28}$ and it has been proposed that TERRA may contribute to telomeric heterochromatin formation by promoting H3K9 trimethylation $^{27,29}$. Recently, we have shown that TERRA is bound by the lysine demethylase LSD1 at TRF2-depleted telomeres, which may promote the physical interaction between LSD1 and MRE11. LSD1 activates MRE11 for the nucleolytic processing of uncapped telomeres ${ }^{28}$. How TERRA transcription is regulated at dysfunctional telomeres and to what extent TERRA may affect chromatin reorganization following the induction of telomeric DDR remains to be elucidated. Here we characterize in detail the transcriptional upregulation of TERRA that ensues telomere deprotection upon depletion of TRF2. We find that
TERRA upregulation is due to increased transcription of telomeres. It occurs independently of ATM-dependent checkpoint signalling and $\mathrm{p} 53$. We demonstrate that TRF2 negatively regulates TERRA transcription through its dimerization domain. We define the TERRA transcriptome and identify conserved transcription factor binding sites that may regulate TERRA transcription. Finally, we provide data supporting a model in which TERRA promotes SUV39H1 binding to TRF2-deficient telomeres and accumulation of $\mathrm{H} 3 \mathrm{~K} 9 \mathrm{me} 3$. Thus, the increased density of $\mathrm{H} 3 \mathrm{~K} 9 \mathrm{me} 3$ at uncapped telomeres may favour telomere end-to-end joining and the activation of Tip60 and the subsequent induction of the DDR pathways.

\section{Results}

TERRA levels increase upon DNA damage and TRF2 loss. To investigate the putative impact of DNA damage on TERRA expression at human telomeres, we monitored TERRA levels in HeLa cells that had been continuously treated with the DSBs generating agent zeocin. FACS profile analysis of zeocin-treated cells showed an accumulation of cells in G2/M (Supplementary Fig. 1a), indicating a prolonged mitotic arrest upon persistent DNA damage. In chromatin immunoprecipitation (ChIP) experiments with antibodies against TRF2, less telomeric DNA was recovered upon zeocin treatment, indicating that reduced amounts of TRF2 remained associated with telomeres (Fig. 1a,b). At the same time ChIP analysis with antibodies against $\gamma$-H2AX indicated that this damage marker accumulated at telomeres (Fig. 1c,d). These results are in agreement with a previous report showing that prolonged mitotic arrest leads to loss of TRF2 from telomeres, telomere deprotection and DDR activation ${ }^{30}$. Next, we measured TERRA levels in time course experiments by northern blot analysis using a strand-specific TERRA probe. Quantification of the hybridization signals revealed that TERRA accumulated at late time points of zeocin treatment over 18S rRNA (Fig. 1e; Supplementary Fig. 1b). To test whether TERRA upregulation was due to telomere deprotection and DDR activation, we monitored TERRA levels following shRNA-mediated depletion of TRF2 and POT1. As expected, shRNA-mediated depletion of TRF2 and POT1 in HeLa cells (Supplementary Fig. 1a) led to the accumulation of $\gamma-\mathrm{H} 2 \mathrm{AX}$ at telomeric but not at centromeric regions as assessed by ChIP using antibodies against $\gamma$-H2AX (Fig. 1f,g). TERRA levels increased upon TRF2 knockdown but they remained unaltered in cells depleted of POT1 (Fig. 1h). Overall, our data suggest that TERRA accumulation upon persistent DNA damage involves depletion of TRF2 from telomeres. To evaluate whether other shelterin proteins may affect TERRA levels, analogous experiments were carried out in HeLa cells depleted for TRF1, Rap1, TIN2 and TPP1 (Supplementary Fig. 2b). As expected for a functional depletion of TRF1, TIN2 and TPP1, telomeric but not centromeric DNA was enriched in ChIP experiments using antibodies against $\gamma$-H2AX (Supplementary Fig. 2c,d). By northern blot analysis, we found that TERRA is also upregulated following TRF1 removal but not upon depletion of the other shelterin components (Supplementary Fig. 2e).

TERRA transcriptome analysis at TRF2-deprotected telomeres. TERRA transcription initiates within subtelomeric regions and proceeds into the telomeric TTAGGG-tracts. TERRA promoters have been identified within $\mathrm{CpG}$ islands that are embedded within repetitive DNA tracts referred to as 61-29-37 repeats ${ }^{25}$. These subtelomeric repeats reside in close proximity of the telomeric TTAGGG-repeats at a large number of chromosome ends ${ }^{25}$. To comprehensively characterize the TERRA 
a

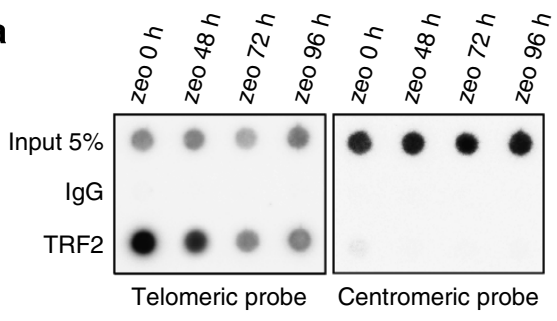

b

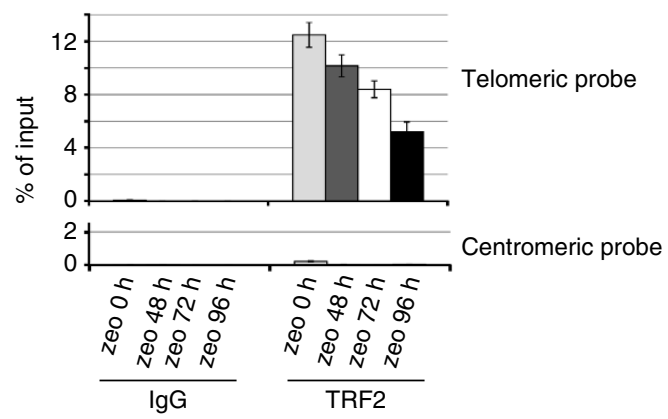

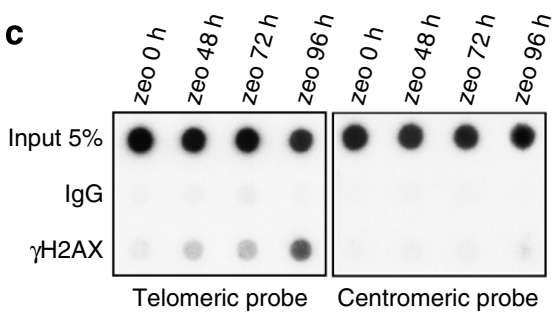

d

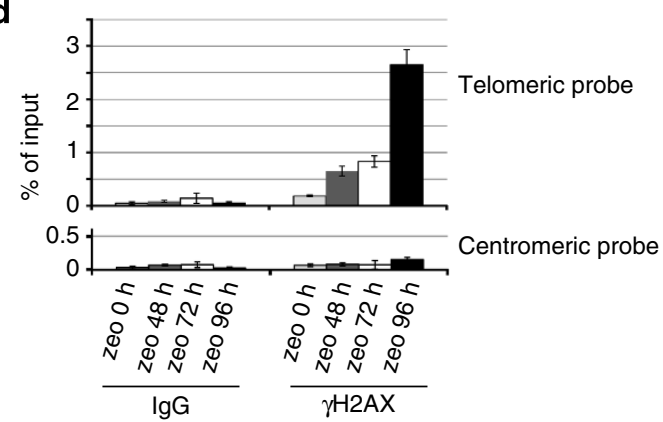

e

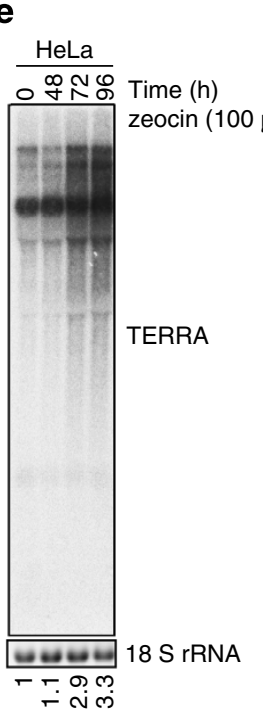

f

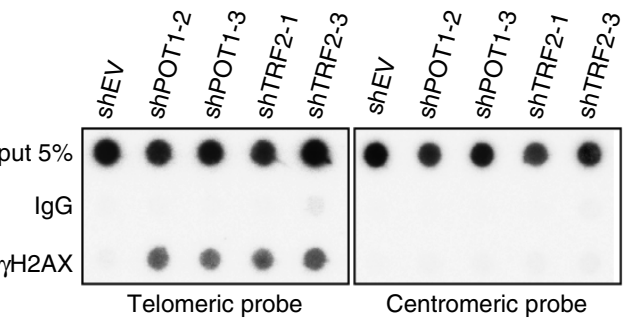

g

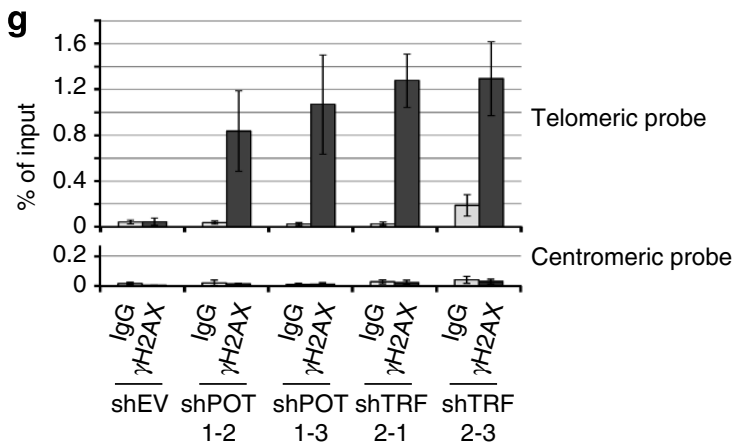

h

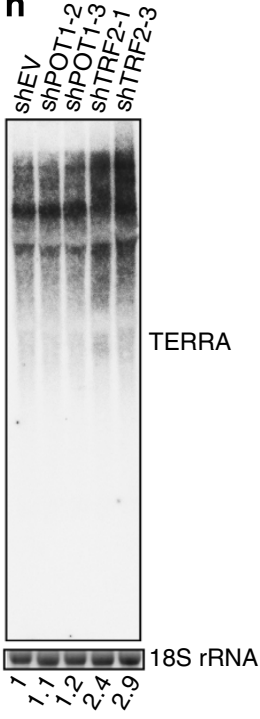

Figure 1 | TERRA expression is upregulated upon persistent DDR and TRF2 depletion. (a) DNA-ChIP of telomeric and centromeric DNA with anti-TRF2 antibody performed in HeLa cells treated with zeocin for the indicated times. (b) Quantification of three independent ChIP experiments represented in a (mean \pm s.d., $n=3$ ). (c) DNA-ChIP of telomeric and centromeric DNA with anti- $\gamma \mathrm{H} 2 \mathrm{AX}$ antibody performed in HeLa cells treated with zeocin for the indicated times. (d) Quantification of three independent ChIP experiments represented in c (mean \pm s.d., $n=3$ ). (e) Northern blot analysis of total RNA from HeLa cells treated with zeocin. TERRA was detected using a telomeric DNA probe complementary to the UUAGGG repeats. Filters were then stripped and reprobed for $18 \mathrm{~S}$ rRNA. (f) DNA-ChIP of telomeric and centromeric DNA with anti- $\gamma \mathrm{H} 2 \mathrm{AX}$ antibody performed in HeLa cells depleted of POT1 and TRF2, respectively. (g) Quantification of three independent ChIP experiments represented in $\mathbf{f}$ (mean \pm s.d., $n=3$ ).

(h) Northern blot analysis of total RNA from HeLa cells depleted of POT1 and TRF2.

transcriptome, we performed high-throughput sequencing using nuclear RNA from wild-type and TRF2-depleted HeLa cells. To enrich for full-length TERRA and minimize ribosomal RNA (rRNA) contamination, we used two purification steps. Since TERRA, but not rRNAs, contains a canonical $m 7 G$ cap $^{28}$, nuclear RNA was first incubated with the high-affinity $m 7 \mathrm{G}$ cap-binding protein eIF4E fused to GST and immobilized on GlutathioneSepharose. The RNA was recovered from the beads and incubated with biotinylated antisense (CCCTAA $)_{3}$ oligonucleotides that specifically annealed to the UUAGGG-tracts residing near the $3^{\prime}$ ends of TERRA transcripts. The RNA bound to biotinylated antisense (CCCTAA) ${ }_{3}$ oligonucleotides was recovered from streptavidin beads. RNA-seq experiments were performed on the TERRA-enriched RNA fraction and on total nuclear RNA (input) from wild-type or TRF2-depleted HeLa cells. The reads were mapped to the most complete assembly available of human subtelomeres (http://www.wistar.org/lab/harold-c-riethman-phd/ page/subtelomere-assemblies) ${ }^{31}$ and read density profiles were generated counting the number of reads aligned at each position along the subtelomeric regions. The TERRA transcripts stemming from different chromosome ends were identified based on their enriched read density in the UUAGGG-containing RNA fraction 

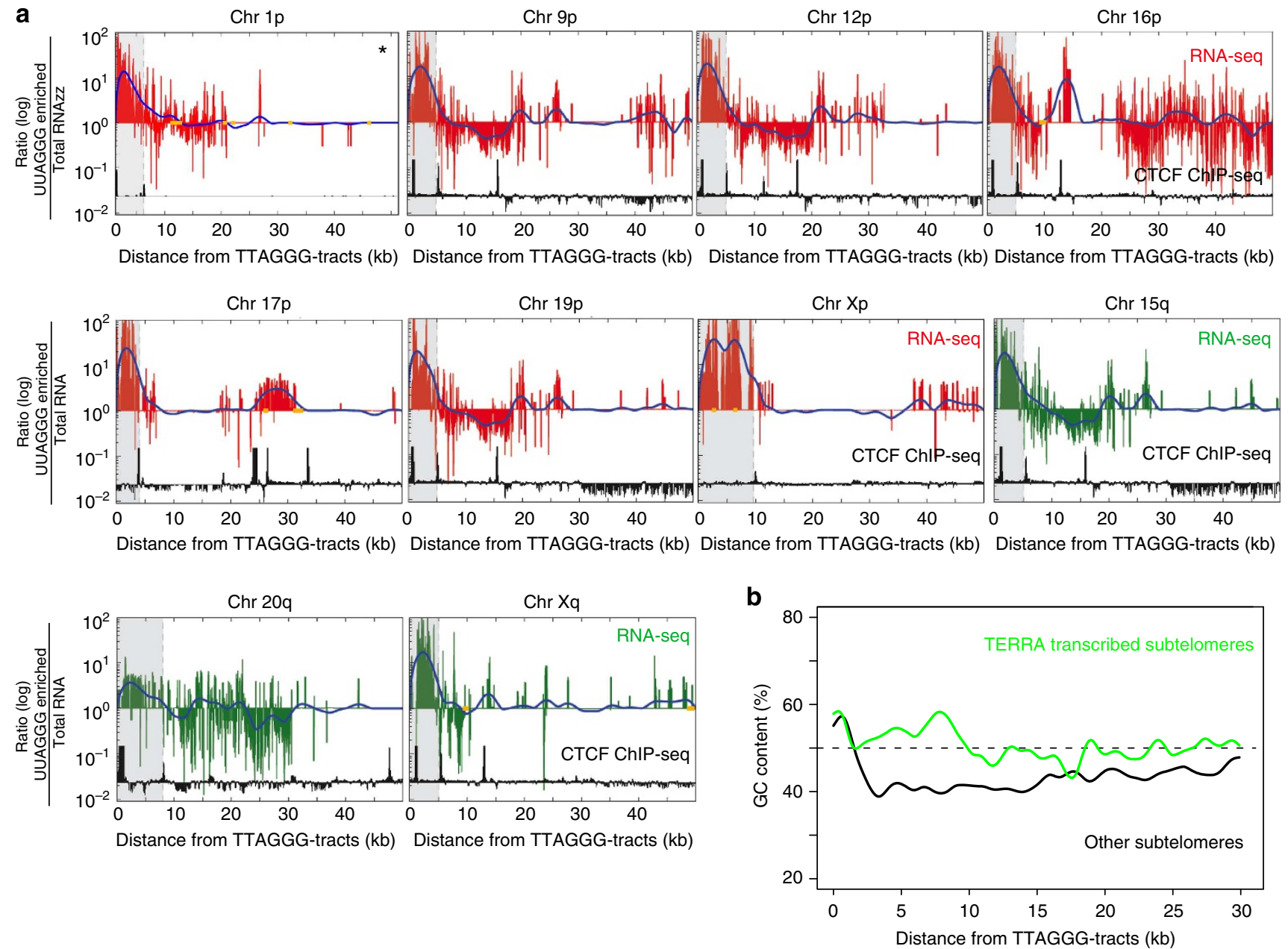

C

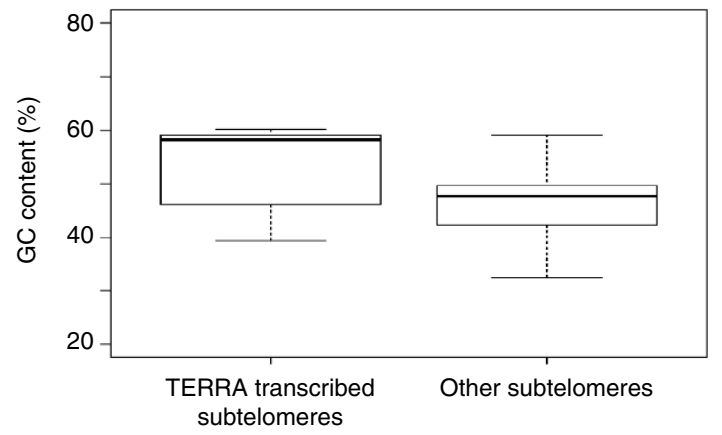

d

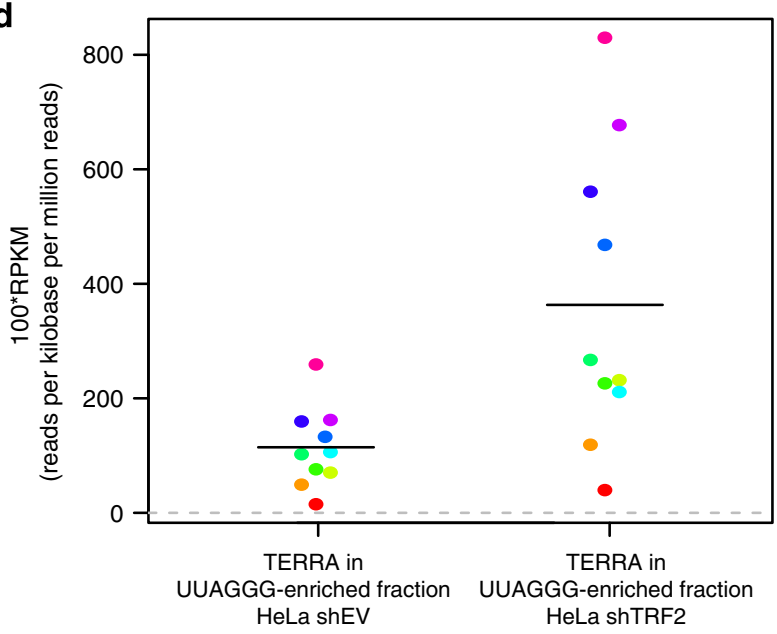

Figure 2 | Characterization of TERRA transcriptome at TRF2-depleted telomeres. (a) Enrichment profiles of RNA-seq data of UUAGGG-containing RNAs versus total nuclear RNA prepared from HeLa cells depleted of TRF2 at 10 different chromosome ends showing transcription within subtelomeric regions. Red and green labelling indicates the $p$ - and the $q$-arms, respectively. Yellow rectangles indicate TTAGGG repeats interspersed in subtelomeric regions. The blue line is a moving average over a window of 10 nucleotides. Enrichment profiles of ChIP-seq analysis of CTCF are shown below the RNA-seq profile data for each chromosome end. (b) Comparison of the GC content of TERRA transcribed versus non-transcribed subtelomeres. The plots were generated using the Wistar GC content track and are an average of the two subtelomere subsets. (c) Average GC content calculated in the first $5 \mathrm{~kb}$ of each subtelomere. The boxes represent the GC content distribution of the two subtelomere subsets (TERRA transcribed versus non-transcribed subtelomeres). The middle line is the median, whereas the lower and upper bounds are the mininimal and maximal values. (d) Reads per kilobase per million reads (RPKM, times $10^{2}$ for practicality) as a measure for chromosome-specific TERRA levels in wild-type (shEV) and TRF2-depleted cells (shTRF2). 
compared with the input material. Doing so, we identified 10 distinct chromosome ends where transcription into TERRA molecules started as far as $5-10 \mathrm{~kb}$ upstream of the subtelomeretelomere boundary (see Fig. 2a for TRF2-depleted HeLa cells and Supplementary Fig. 3 for wild-type HeLa cells). At eight other chromosome ends, the RNA-seq data suggested that transcription starts in close proximity to the TTAGGG-tracts (Supplementary Fig. 4). Despite good mappability scores for several additional chromosome ends, the remaining complete subtelomeres did not display consistent read profiles in the subtelomeric regions (Supplementary Fig. 5). However, due to the repetitive nature of the human telomeric repeat sequences, the reads containing only UUAGGG repeats could not be assigned to a specific chromosome end. Thus, we cannot rule out that transcription at several chromosome termini may initiate within the terminal TTAGGG-repeat sequences. Our RNA-seq data also revealed that TERRA $5^{\prime}$ ends are embedded within subtelomeric regions with higher GC content than the non-transcribed subtelomeres (Fig. 2b). In addition, using published ChIP-seq data from
Deng et al. ${ }^{32}$, we ascertain that CTCF, which positively regulates TERRA transcription, binds subtelomeres in the vicinity of TERRA $5^{\prime}$ ends (Fig. 2a; Supplementary Fig. 4).

Next, we identified cis-regulatory elements and transcription factors (TFs) that may control TERRA expression. To this aim, we used TRANSFAC databases and scanned for transcriptional regulatory modules in the subtelomeric regions flanking the identified TERRA transcription start sites. For the TERRA promoters located $5-10 \mathrm{~kb}$ upstream of the telomeric tracts, our analysis revealed an overrepresentation of binding sites for zinc finger transcription factors (ZFP281, ZFP740 and ZFX) and members of the SP/KLF (specificity protein/Kruppel-like factor) family, such as KLF15, SP1, SP2 and SP4 (Supplementary Table 1). On the other hand, the TERRA promoters located in close proximity of the terminal TTAGGG-repeat sequences displayed an overrepresentation of sequence motifs that can be bound by interferon regulatory factors, basic helix-loop-helix transcription factors, such as myogenic regulatory factors $(\mathrm{MyoD}$, Myf, Myogenin) and GATA-type zinc finger transcription factors

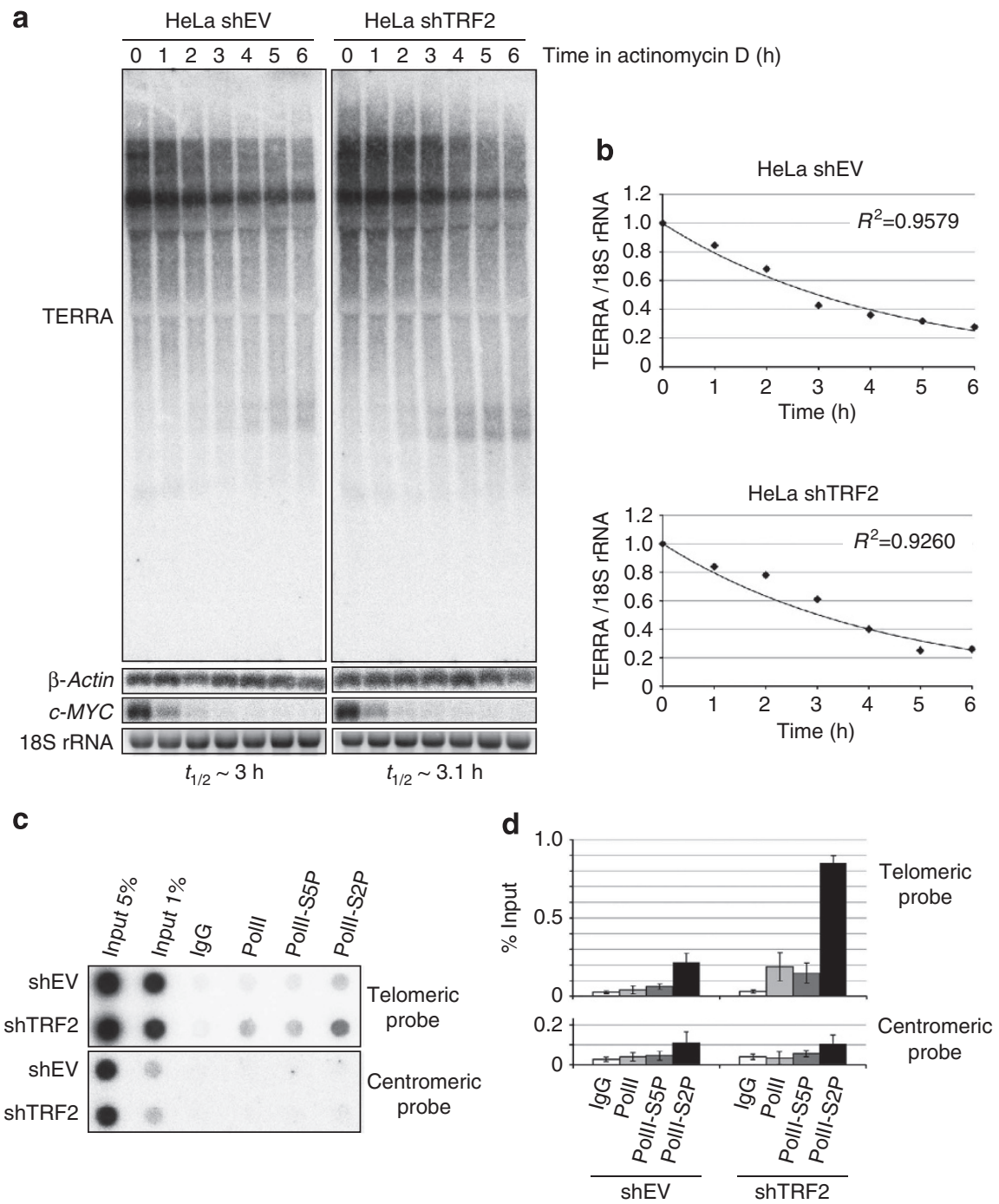

Figure 3 | TERRA transcriptional rate but not half-life increases upon TRF2 depletion. (a) TERRA half-life. Wild-type and TRF2-depleted HeLa cells were treated with $5 \mu \mathrm{g} \mathrm{ml}^{-1}$ actinomycin D for the indicated times. Total RNA was prepared and subjected to Northern blot analysis using a telomeric DNA probe detecting UUAGGG repeats. Filters were then stripped and reprobed successively for c-MYC, $\beta$-actin and 18S rRNA. (b) Quantification of TERRA half-life in wild-type and TRF2-depleted HeLa cells based on northern blots shown in a. Relative TERRA levels were calculated using a scatter plot analysis after normalizing TERRA to $18 \mathrm{~S}$ rRNA. The best-fit exponential regression curve of the data points was determined for both conditions. $R^{2}$ values are indicated. (c) DNA-ChIP of telomeric and centromeric DNA with anti-RNAPII antibodies performed in HeLa cells depleted of TRF2.

(d) Quantification of three independent ChIP experiments represented in c (mean \pm s.d., $n=3$ ). 
(GATA1 and GATA5) (Supplementary Table 2). The binding motif for the transcriptional factor CTCF is overrepresented at both the telomere distal and proximal TERRA promoters (Supplementary Tables 1 and 2), thus further supporting the role of CTCF in regulating TERRA expression.

Finally, we compared the abundance of the different TERRA molecules that contained long subtelomeric sequences by calculating the number of reads per kilobase per million reads $(\mathrm{RPKM})^{33}$ in the region between the TERRA $5^{\prime}$ ends and the start of the telomeric tract $(5-10 \mathrm{~kb}$; not spliced; Supplementary Table 3). The abundance of TERRA RNAs from the different chromosome ends was in the same bulk part and TERRA abundance increased similarly at all measured chromosome ends 2 to 4 -fold upon depletion of TRF2 confirming the previous results (Fig. 2d and Supplementary Table 3). The TERRA RPKMs were compared with the values obtained for four different housekeeping genes. TERRA abundance from individual chromosomes ends was 10 times lower than transcripts from the GUSB gene (encoding for $\beta$-glucuronidase) and 100 to 1,000 times lower than the transcripts of three other highly expressed housekeeping genes (GAPDH, ACTB and TUBA1) (Supplementary Table 3).

Increased transcription in TRF2-deficient cells. TERRA upregulation at deprotected telomeres could be a result of a longer half-life of the transcripts or an increased transcription rate. To distinguish between these two possibilities, we first measured TERRA half-life. We blocked transcription in wild-type and TRF2-depleted HeLa cells with actinomycin D and monitored TERRA levels in time course experiment by northern blot analysis. $\beta$-Actin, $c-$ Myc and $18 \mathrm{~S}$ rRNA were included in the analysis for normalization ( $\beta$-actin and $18 \mathrm{~S}$ rRNA) and to control for successful inhibition of RNAPII (c-Myc) $((t 1 / 2)$ of $20 \mathrm{~min}$ (refs 34-36); Fig. 3a). Quantification of the hybridization signals revealed that, as previously shown ${ }^{23,28}$, total TERRA has a halflife of $\sim 3 \mathrm{~h}$ in wild-type cells. In TRF2-depleted cells, the half-life increased only marginally to $\sim 3.1 \mathrm{~h}$ upon TRF2 removal (Fig. 3b), which cannot account for the accumulation of TERRA transcripts in TRF2-deficient cells.

To obtain clues about transcriptional activity at wild-type and TRF2-depleted telomeres, we performed ChIP experiments using antibodies against RNA polymerase II (RNAPII) and its phosphorylated forms (Fig. 3c,d). Telomeric DNA was significantly enriched in TRF2-depleted cells over wild-type cells with all RNAPII antibodies. Most striking was the difference with an antibody against the elongating form of RNAPII recognizing the phosphorylated serine in the amino acid 2 position of the YSPTSPS-repeat sequence in the carboxy (C)-terminal domain of RNAPII (PolII-S2P in Fig. 3c,d). Together, these experiments indicate that TERRA accumulation upon telomere deprotection is not because of an increase in the stability of the transcript but is caused by a higher transcription rate.

Damage signalling is not required for TERRA upregulation. To assess the influence of DNA damage signalling on the increased levels of TERRA upon TRF2 knockdown, we depleted separately MRE11 or NBS1, two subunits of the MRN complex, and the ATM and ATR kinases by shRNA. MRE11 or NBS1 were efficiently depleted in wild-type and TRF2-deficient HeLa cells as determined by western blot analyses (Fig. 4a). Furthermore, a reduction of telomere end-to-end fusions in the TRF2-depleted cells indicated loss of function of MRE11 and NBS1 (Supplementary Fig. 6a). TERRA levels were monitored by northern blot analysis and quantitative RT-PCR. Depletion of the MRN complex components neither affected TERRA levels in wild-type cells nor did it prevent TERRA upregulation at TRF2-deprotected telomeres (Fig. 4b; Supplementary Fig. 6e). Similar experiments were performed in wild-type and TRF2deficient cells efficiently depleted of ATM and ATR (Fig. 4c). The depletions of ATM and ATR both were functional as phosphorylation of the downstream Chk2 and Chk1 kinases was reduced (Supplementary Fig. 6b). Functional depletion of ATM was further confirmed in ChIP experiments in which $\gamma$-H2AX no longer accumulated at telomeres in TRF2-deficient cells (Supplementary Fig. 6c,d). Analogous to what was observed for the MRN complex, depletion of ATM and ATR had no effect on TERRA levels at both functional and deprotected telomeres (Fig. 4d; Supplementary Fig. 6f). In addition to ATM and ATR, we depleted SMG1, which is also a member of the PI3K-related kinase (PIKK) family. SMG1 has well-characterized roles in nonsense-mediated RNA decay and in the $\mathrm{DDR}^{37,38}$. In addition, SMG1 negatively regulates the abundance of TERRA at telomeres $^{23}$. We knocked down SMG1 with two different shRNAs alone or in combination with TRF2 (Supplementary Fig. 7a), which concomitantly reduced phosphorylation of UPF1 as expected for loss function of SMG1 (Supplementary Fig. 7b) and evaluated TERRA levels by quantitative RT-PCR. No significant difference in TERRA levels was observed upon SMG1 depletion in both wild-type and TRF2-deficient cells (Supplementary Fig. 7c). Finally, we tested if p53 may control TERRA induction upon telomere deprotection. For this, we employed two isogenic human colorectal carcinoma cell lines (HCT116), which were wild-type $(\mathrm{p} 53+/+)$ or knocked out for p53 (p53-I- ; ref. 39). We transfected the p53 ${ }^{+/+}$and the p53-7- cells with shRNAs against POT1 or TRF2 and we found that TERRA upregulation occurred upon TRF2 but not POT1 removal in presence and absence of p53 (Fig. 4e). Thus p53 is dispensable for TERRA transcription at wild-type and TRF2deprotected telomeres. Overall, these results strongly suggest that DDR activation does not account for TERRA upregulation following telomere deprotection by TRF2. Therefore, we postulated that TRF2 might act directly as a negative regulator of TERRA transcription.

The homodimerization domain of TRF2 represses TERRA. TRF2 and TRF1 both consist of four domains: a C-terminal MYB domain required for binding to telomeres, a flexible hinge domain involved in protein-protein interactions, a TRFH domain required for homodimerization ${ }^{40}$ and chromatin compaction ${ }^{41,42}$, and a divergent amino $(\mathrm{N})$-terminal region rich in basic amino acids in TRF2 and acidic residues in TRF1. To identify the molecular properties of TRF2 involved in regulating TERRA transcription, we swapped, inspired by a previous study ${ }^{41}$, several domains between TRF2 and TRF1 (refs 43,44) in cDNA constructs (Fig. 5a) and expressed them in HeLa cells (Fig. 5b). Western blot analysis and IF-FISH experiments indicated that the TRF2/TRF1 chimeric proteins were expressed at comparable levels (Fig. 5b) and that they localized to telomeres even though the nucleoplasmic patterns differed slightly with the different constructs (Fig. $5 \mathrm{c}$ ). We then tested whether the TRF2 chimeric constructs were able to prevent induction of TERRA transcription in HeLa cell lines depleted of endogenous TRF2 by shRNA. Importantly, ectopically expressed full-length wild-type TRF2 but not TRF1 prevented TERRA upregulation upon depletion of endogenous TRF2 (Fig. 5d). This suggests that TRF2 and TRF1 suppress TERRA abundance through distinct mechanisms. The TRF2 construct lacking the N-terminal basic region (TRF2- $\Delta \mathrm{B}$ ) was also able to suppress the transcriptional induction of TERRA that followed loss of endogenous TRF2 (Fig. 5d) ruling out a critical and specific function of this domain in TRF2. Similarly, 

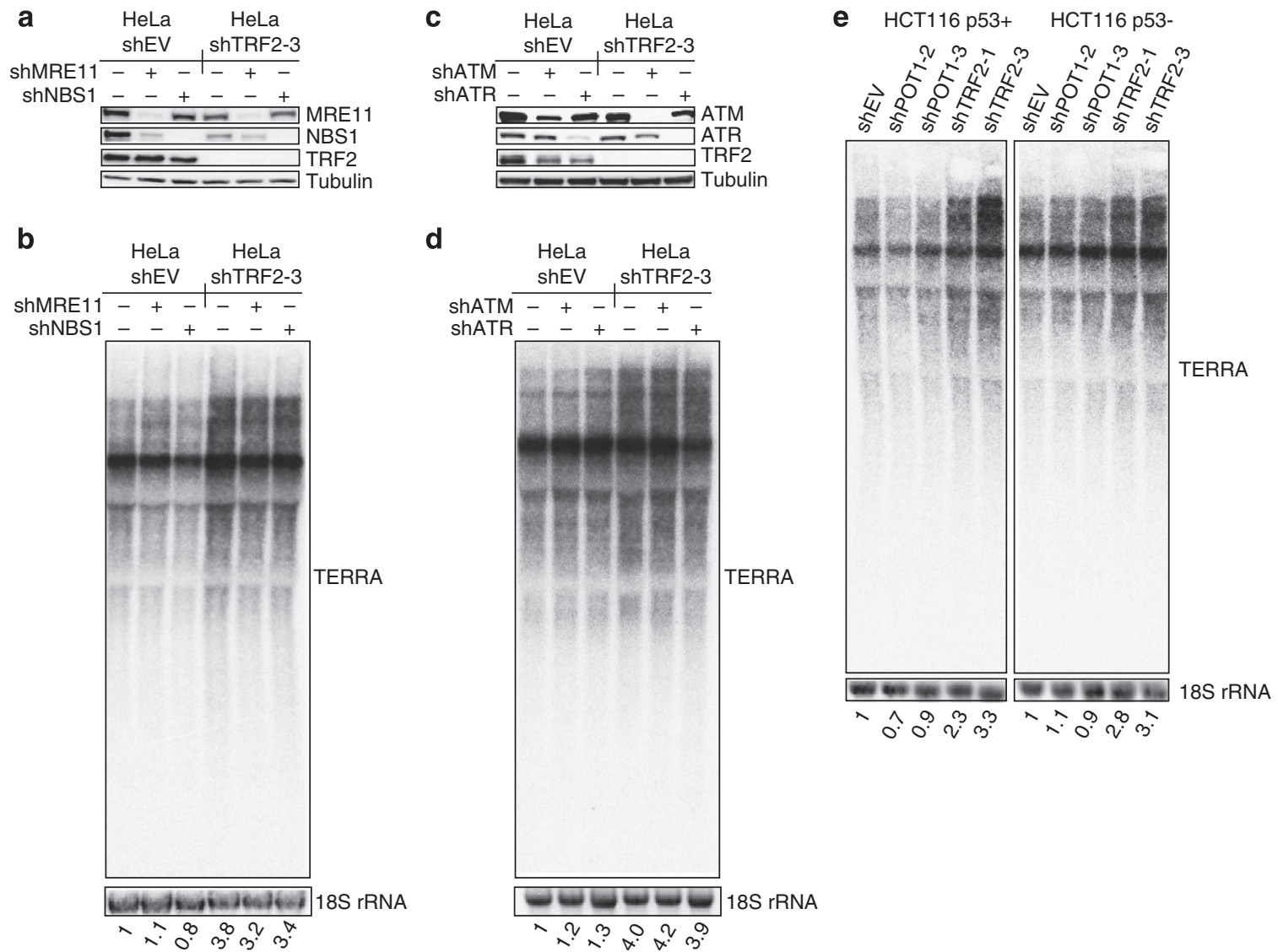

Figure 4 | DDR activation is not required for TERRA upregulation at uncapped telomeres. (a) Western blot analysis to evaluate knockdown efficiency of endogenous MRE11, NBS1 and TRF2. Tubulin was used as loading control. (b) Wild-type (shEV) and TRF2-depleted (shTRF2) HeLa cells transfected with control vector (shEV) or additionally depleted for MRE11 (shMRE11) and NBS1 (shNBS1). Total RNA was prepared and subjected to northern blot analysis using a telomeric DNA probe detecting UUAGGG repeats. Filters were then stripped and reprobed subsequently for $18 \mathrm{~S}$ rRNA. (c) Western blot analysis to evaluate knockdown efficiency of endogenous ATM, ATR and TRF2. Tubulin was used as loading control. (d) Wild-type (shEV) and TRF2-deficient (shTRF2) HeLa cells transfected with control vector (shEV) or depleted for ATM (shATM) and ATR (shATR). Total RNA was prepared and subjected to northern blot analysis using a telomeric DNA probe detecting UUAGGG repeats. Filters were then stripped and reprobed subsequently for $18 \mathrm{~S}$ rRNA. (e) Northern blot analysis of total RNA prepared wild-type (p53+) and p53 knockout (p53-) HCT116 cells. The blot was probed using a telomeric DNA probe detecting UUAGGG repeats. The filter was stripped and reprobed subsequently for 18S rRNA. Uncropped western blots are shown in Supplementary Fig. 9a,b.

the hinge and DNA binding domains of TRF2 also did not seem to have critical roles for the TRF2-specific repression of TERRA as they could be replaced with the corresponding domains from TRF1 (TRF2- $\Delta \mathrm{BcH}$ and TRF2- $\Delta \mathrm{BcM}$ in Fig. 5). In contrast, ectopic expression of the TRF2 chimeric protein containing the TRFH domain of TRF1 (TRF2- $\Delta \mathrm{BcT}$ ) did not prevent TERRA accumulation in the absence of endogenous TRF2 (Fig. 5d). Overall, these data suggest that TRF2 represses TERRA transcription through its TRFH domain. This domain may elicit this function through its capacity in structural compaction of chromatin ${ }^{41,42}$, making telomeres less accessible to RNAPII. Interestingly, it has recently been shown that the TRFH domain of TRF2 is also required to prevent the activation of the ATM pathway and the initial steps in the telomeric $\mathrm{DDR}^{44}$.

TERRA upregulation may sustain H3K9me3 accumulation. At telomeres, it has been suggested that TERRA may participate in the accumulation of H3K9me3 (refs 27,29). We investigated by ChIP the histone methylation pattern upon telomere uncapping. The density of monomethylated H3K4 was not affected following TRF2 depletion and the signals for di- and trimethylated H3K4 were close to background at both, capped and unprotected telomeres (Supplementary Fig. 8a,b). Also monomethyl H3K9 was not affected at telomeres following TRF2 depletion (Fig. 6a). However, we observed a significant reduction of dimethylated $\mathrm{H} 3 \mathrm{~K} 9$ (H3K9me2) and a corresponding rise in the density of H3K9me3 upon telomere uncapping, whereas they both remained unaltered at centromeric chromatin (Fig. 6a,b). At DSBs occurring in heterochromatic regions, $\mathrm{H} 3 \mathrm{~K} 9 \mathrm{me} 3$ provides activation sites for Tip60 (KAT5) (ref. 10), which affects H4K16 acetylation and ATM activation. In line with this, we observed that TRF2-deprotected telomeres showed an increase in H4K16 acetylation, which is indicative of Tip60 activity (Supplementary Fig. 8c). We tested whether the accumulation of $\mathrm{H} 3 \mathrm{~K} 9 \mathrm{me} 3$ at dysfunctional telomeres coincides with recruitment of the histone methyltransferase SUV39H1. We performed ChIP experiments with two different antibodies against endogenous SUV39H1 and found that the telomeric signal in the SUV39H1-ChIP significantly increased upon telomere uncapping by TRF2 depletion (Fig. $6 c, d$ ) suggesting that this protein is responsible for $\mathrm{H} 3 \mathrm{~K} 9$ trimethylation at TRF2-depleted telomeres. Next, we assessed whether SUV39H1 can interact with TERRA. To this aim, we performed RNA immunoprecipitation experiments with extracts derived from formaldehyde-crosslinked HeLa cells followed by stringent washing conditions. Approximately $1 \%$ of TERRA was immunoprecipitated with two different antibodies 
a
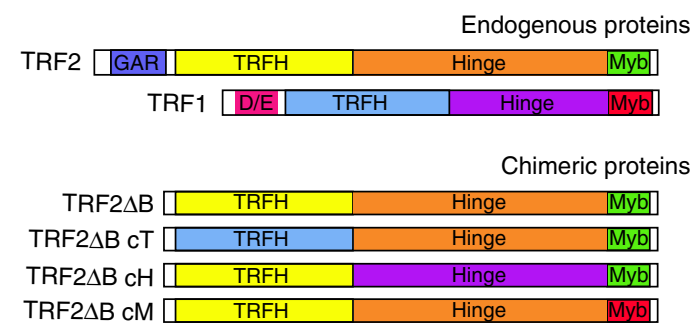

b

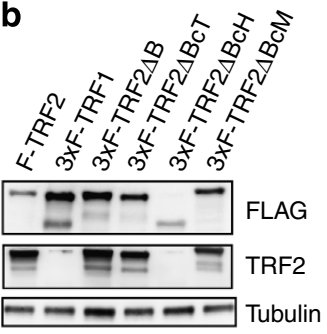

C

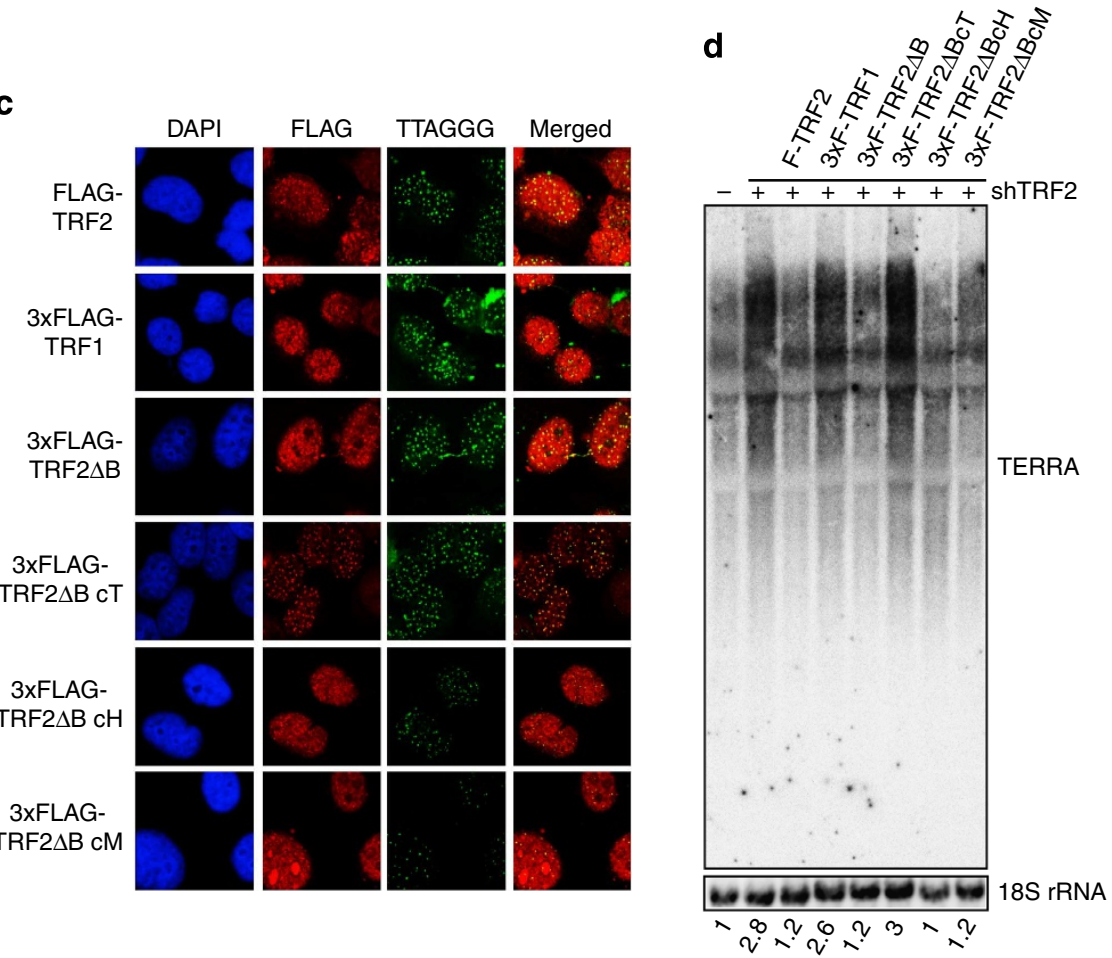

Figure 5 | TRF2 negatively regulates TERRA transcription through its homodimerization TRFH domain. (a) Schematic representation of TRF2/TRF1 chimeric constructs. (b) Western blot analysis to test expression of TRF2/TRF1 chimeric proteins in HeLa cells depleted of endogenous TRF2. Uncropped blots are shown in Supplementary Fig. 9c. (c) Immunofluorescence fluorescence in situ hybridization (IF-FISH) analysis to assess the localization of the TRF2/TRF1 chimeric proteins (red signals) at telomeres (green signals) in HeLa cells depleted of endogenous TRF2. (d) Northern blot analysis. Total RNA was prepared from wild-type HeLa cells or HeLa cells depleted of TRF2 that had been transfected with the indicated constructs. Northern blot analysis was then performed using a telomeric DNA probe detecting UUAGGG repeats. Filters were then stripped and reprobed subsequently for $18 \mathrm{~S}$ rRNA.

against endogenous SUV39H1 upon telomere uncapping. In contrast, this interaction was barely detectable in cells with normal levels of TRF2. Thus the number of TERRA-SUV39H1 complexes increased substantially upon TRF2 removal (Fig. 6e). Remarkably, TERRA was also significantly enriched upon H3K9me3 RNA-IP of formaldehyde-crosslinked extracts following telomere deprotection indicating that TERRA, SUVH39H1 and $\mathrm{H} 3 \mathrm{~K} 9 \mathrm{me} 3$ may be present in common structures. The specificity of these interactions was confirmed by the fact that TERRA did not immunoprecipitate with IgG control. 18S rRNA served as negative control and was not retrieved by SUV39H1 or $\mathrm{H} 3 \mathrm{~K} 9 \mathrm{me} 3$ immunoprecipitation. Together, these experiments indicate that TERRA upregulation may account for the increased recruitment of SUV39H1 to uncapped telomeres and the subsequent accumulation of $\mathrm{H} 3 \mathrm{~K} 9 \mathrm{me} 3$ upon depletion of TRF2.

SUV39H1 N-terminus binds the UUAGGG repeats of TERRA. To assess whether SUV39H1 binds TERRA directly, the biochemical properties of recombinant GST-SUV39H1 were characterized in electromobility shift assays (EMSAs). GST-SUV39H1 was expressed in E. coli, purified (Fig. 7a) and incubated with labelled TERRA-mimicking RNA oligonucleotide

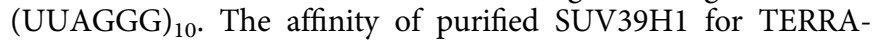
mimicking RNA oligonucleotides was shown by the retarded migration of the labelled oligonucleotide (Fig. 7b) and the identity of the shifted complex was confirmed in supershift mobility assays with the use of an antibody recognizing GST-SUV39H1 (Fig. 7c). In contrast to the TERRA-oligonucleotide, labelled DNA of the same sequence (TTAGGG) 10 was not bound by SUV39H1 (Fig. 7b). Further, the binding specificity was addressed by EMSA in competition experiments in which the associa-

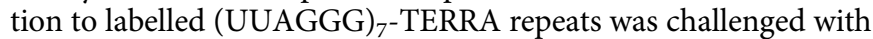
excess of unlabelled wild-type and mutant telomeric repeats (Fig. 7d). This analysis indicated that SUV39H1 has specificity for the AGGG sequence within the UUAGGG-repeats but it does not recognize the UU-dinucleotide (Fig. 7d). Finally, we delineated the TERRA interaction domains of SUV39H1. Two bacterially expressed GST-SUV39H1 fragments were purified (Fig. 7e,f) and characterized by EMSA for their ability to bind TERRA- 
a

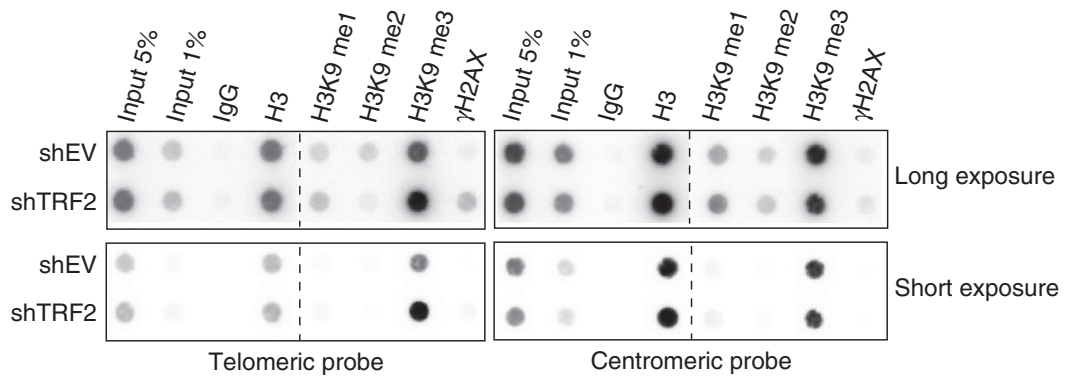

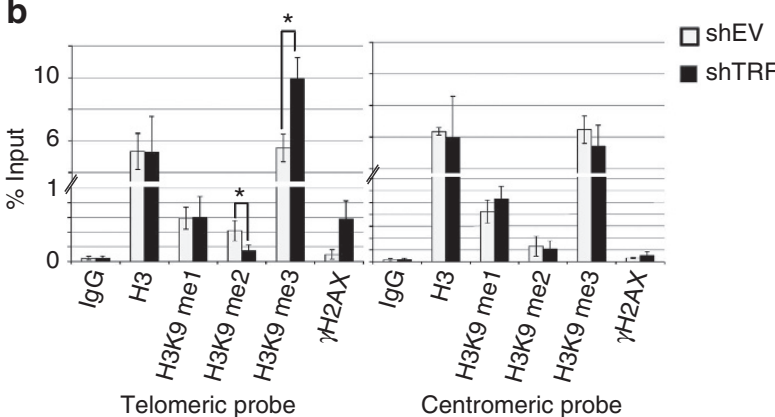

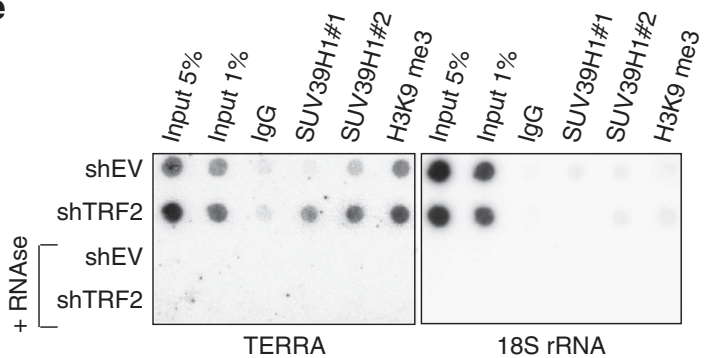

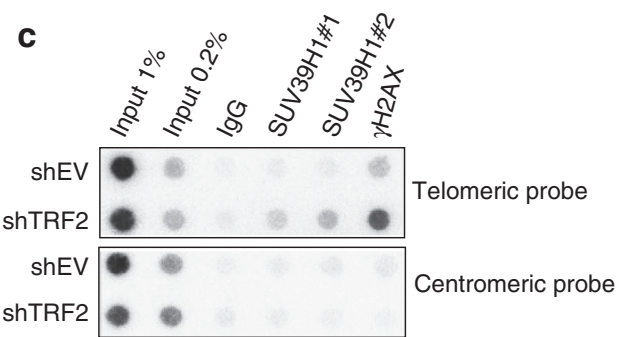

d $0.8 \longrightarrow$ shEV

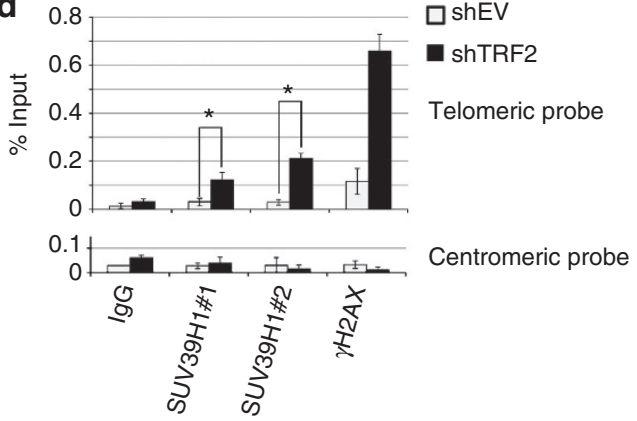

Figure 6 | SUV39H1 associates with TERRA and is recruited to uncapped telomeres. (a) ChIP of telomeric and centromeric DNA with H3 antibodies recognizing histone marks in HeLa cells transfected with control vector (shEV) or depleted of TRF2 (shTRF2). (b) Quantification of three independent ChIP experiments represented in a (mean \pm s.d., $n=3$ ). Statistical analysis was done using a two-tailed Student's $t$-test $\left({ }^{\star} P<0.05\right)$. (c) ChIP of telomeric and centromeric DNA with anti-SUV39H1 antibodies performed in HeLa cells transfected with control vector (shEV) or depleted of TRF2 (shTRF2). (d) Quantification of three different ChIP experiments represented in c (mean \pm s.d., $n=3$ ). Statistical analysis was done using a two-tailed Student's t-test $\left({ }^{\star} P<0.05\right)$. (e) RNA-ChIP assays with two antibodies against endogenous SUV39H1 performed in HeLa cells transfected with control vector (shEV) or depleted of TRF2 (shTRF2). IP-recovered RNA was detected with probes annealing with TERRA (left panel) or 18S rRNA (right panel).

mimicking RNA oligonucleotides (UUAGGG) 10 . Only the $\mathrm{N}$-terminal SUV39H1 fragment encompassing the chromodomain was able to substantially retard the migration of the labelled TERRA-mimicking oligonucleotide (Fig. 7g) with a dissociation constant for (UUAGGG) 10 of $\sim 80 \mathrm{nM}$. In summary, we conclude that SUV39H1 can bind directly via its N-terminal chromodomain the UUAGGG-repeat array of the TERRA transcript, with moderate specificity.

To assess the roles of SUV39H1 in telomere end-to-end fusions at TRF2-depleted telomeres, we used a HeLa cell line in which TRF2 can be depleted by doxycycline-inducible expression of shRNAs ${ }^{45}$ (Fig. 8a). Significantly, depletion of SUV39H1 by siRNAs in the TRF2-depleted cell line (Fig. 8b) reduced telomere end-to-end fusion events (Fig. $8 \mathrm{c}$ and Supplementary Table 4) indicating that the chromatin modifications induced by this enzyme sustain end-to-end fusions in accordance with data obtained in mouse cells upon deletion of SUV39H1 (ref. 46).

\section{Discussion}

In this study, we characterize the transcriptional response of telomeres that follows uncapping upon depletion of TRF2. Our data show that damaged telomeres are transcriptionally derepressed which is typical for damaged heterochromatic domains. Using high-throughput RNA-seq, we also characterize the whole
TERRA transcriptome. Our results demonstrate that transcription initiation of TERRA at different chromosome ends varies substantially with regard to its position to the telomeric repeat tract extending in some cases for several kilobases into the subtelomeric regions. This observation is in agreement with a broad distribution of RNAPII across subtelomeres that was reported previously ${ }^{32}$. Analysis of the subtelomeric sequence reads did not reveal any splicing events of TERRA transcripts suggesting that TERRA transcripts in contrast to many other long noncoding RNAs are not spliced. Sequences with enriched GC content located from $5-10 \mathrm{~kb}$ upstream of the telomeric tracts may function as promoters and drive TERRA transcription near at least 10 different chromosome ends. On the other hand, the previously reported set of $\mathrm{CpG}$ island promoters ${ }^{25}$ appear responsible for driving the transcription of the TERRA molecules whose $5^{\prime}$ ends localize in close proximity to the telomeric tracts. Overall, the promoter distribution of TERRA at different chromosome ends can account for the length heterogeneity of the transcripts. We cannot rule out the possibility that transcription may also start within telomeric repeats or within immediate proximity thereof, leading us to underestimate the real number of transcribed chromosome ends; similarly, we cannot rule out cell line-specific effects that could impact the distribution of TERRA-expressing telomeres. We 


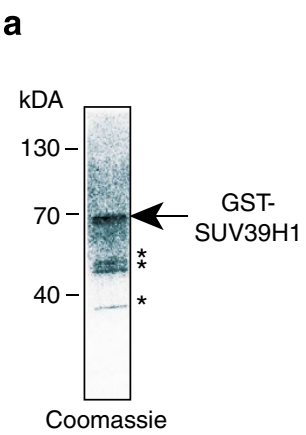

b
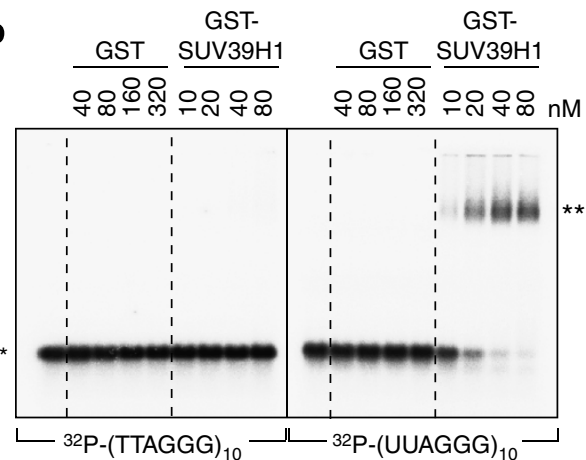

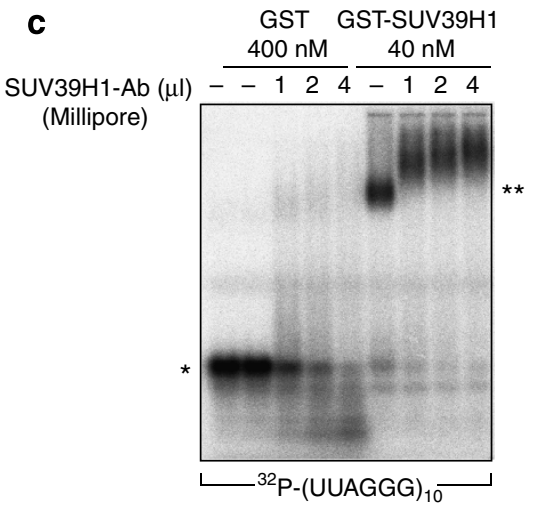

d

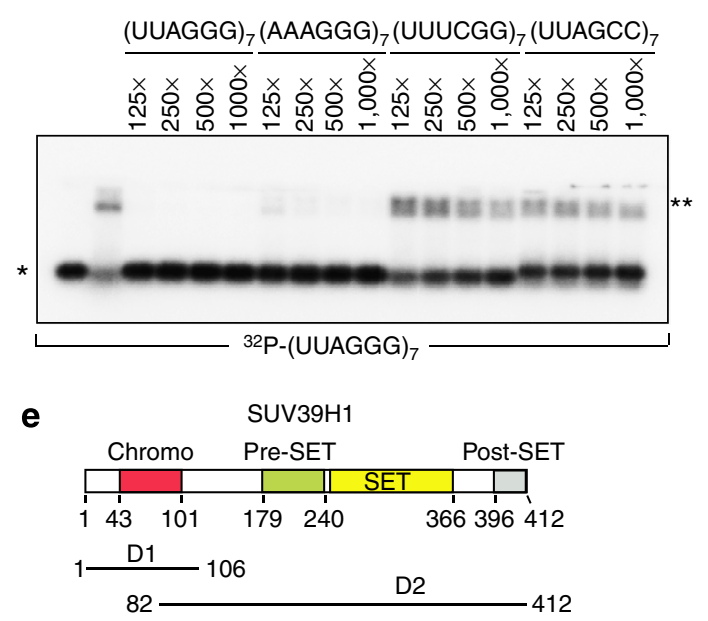

f

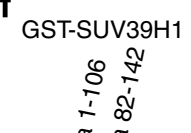

(2)

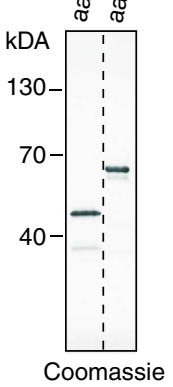

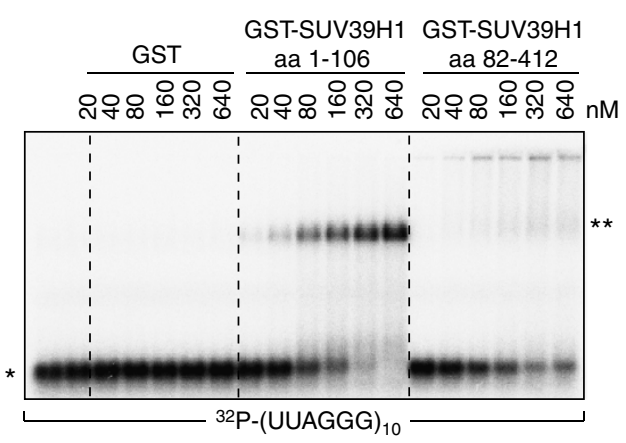

Figure 7 | The N-terminal domain of SUV39H1 directly binds UUAGGG-TERRA repeats. (a) Coomassie stained gel of GST-SUV39H1 affinity purified from E. coli. Bands labelled with asterisks are SUV39H1 degradation products. (b) Agarose EMSA used to measure SUV39H1 RNA binding activity in vitro. GST-SUV39H1 was assayed for binding to ${ }^{32} \mathrm{P}-(\mathrm{UUAGGG})_{10}$ RNA or ${ }^{32} \mathrm{P}-($ TTAGGG) 10 DNA oligonucleotides, respectively. Single asterisks indicate free oligonucleotides and double asterisks protein/oligonucleotide complexes. (c) Agarose supershift assay performed with GST-SUV39H1 incubated with ${ }^{32} \mathrm{P}-(\mathrm{UUAGGG})_{10}$ RNA oligonucleotide and an antibody recognizing SUV39H1 polypeptide. Single asterisks indicate free oligonucleotides and double asterisks protein/oligonucleotide complexes. (d) EMSA competition experiments of GST-SUV39H1 binding to ${ }^{32}$ P-(UUAGGG). Increasing amounts of unlabeled (UUAGGG) 7 , (AAAGGG) ${ }_{7}$ (UUUCGG) $)_{7}$ and (UUAGCC) $)_{7}$ RNA oligonucleotides were added as competitors. Numbers indicate fold excess of competitor over ${ }^{32} \mathrm{p}$-labelled probe. Single asterisks indicate free oligonucleotides and double asterisks protein/oligonucleotide complexes.

(e) Scheme of SUV39H1 domains and deletion constructs. (f) Coomassie stained gel of GST-SUV39H1 deletion constructs affinity purified from E. coli. (g) Agarose EMSA used to identify domains of SUV39H1 with binding activity for ${ }^{32} \mathrm{P}$-(UUAGGG) 10 RNA oligonucleotides. Single asterisks indicate free oligonucleotides and double asterisks protein/oligonucleotide complexes.

provide a bioinformatic analysis of the putative TERRA promoters and identify transcription factor modules that might regulate TERRA. Among the putative TERRA transcription factors, we find that CTCF, previously shown to regulate TERRA expression ${ }^{32}$, not only localizes near the $5^{\prime}$ ends of the CPG island TERRA promoters but also to the more telomere distal TERRA promoters identified in our analysis.

Despite a previous study that reported a p53-dependent increase in TERRA levels at damaged telomeres ${ }^{47}$, we provide evidence that TERRA induction at TRF2-depleted telomeres occurs independently of p53 and does not require the ATM- or ATR-dependent DNA damage signalling cascade. Therefore, although ATM can silence transcription in the vicinity of DSBs occurring in euchromatic regions to prevent chromatin movement and maintain proximity of broken termini ${ }^{3}$, it does not regulate transcription at telomeres. On the other hand, we observe that TRF2 directly suppresses TERRA transcription through its homodimerization domain. The TRFH domain of TRF2 has been implicated in chromatin compaction ${ }^{41,42}$ and it might be involved in sequestering chromosome termini within the T-loop structure ${ }^{48,49}$, which may protect the ends of chromosomes from the DNA damage machinery. When TRF2 is removed, the higher order telomere structures are disrupted and the telomeric chromatin becomes accessible to RNAPII. Moreover, the TRFH domain of TRF2 is required to prevent the activation of ATM and the initial steps in the DDR pathway ${ }^{44}$. This observation together with the evidence that the DDR pathways are not responsible for TERRA accumulation, lead us to hypothesize that TERRA may function either upstream or in parallel to ATM signalling upon telomere deprotection.

What is then the role of TERRA upregulation at uncapped telomeres? In addition to its roles in telomere end processing ${ }^{50}$, we propose a functional link between TERRA induction and chromatin reorganization of dysfunctional telomeres. TERRA has been implicated in the accumulation of $\mathrm{H} 3 \mathrm{~K} 9 \mathrm{me} 3$ at functional telomeres facilitating heterochromatin formation ${ }^{27,29}$. Moreover, at elongated telomeres increased density of $\mathrm{H} 3 \mathrm{~K} 9 \mathrm{me} 3$ correlates with an increase in TERRA-derived UUAGGG signal ${ }^{27}$. Here, we show that the UUAGGG-tract of TERRA interacts with the $\mathrm{N}$-terminal domain of the histone methyltransferase SUV39H1 
a

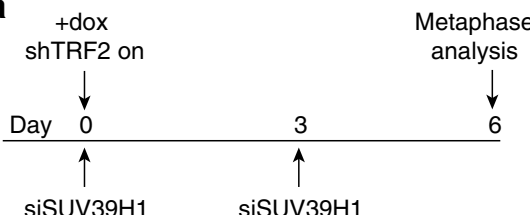

C
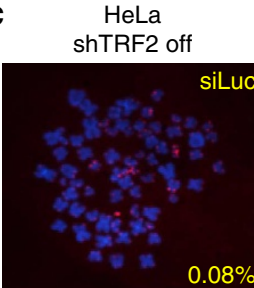

$0.08 \%$

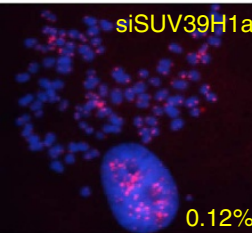

$0.12 \%$

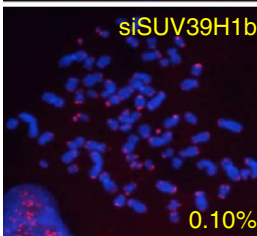

HeLa shTRF2 on

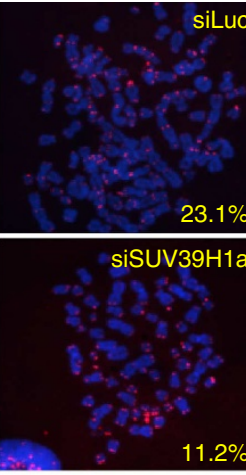

siSUV39H1b

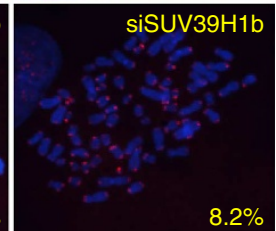

b

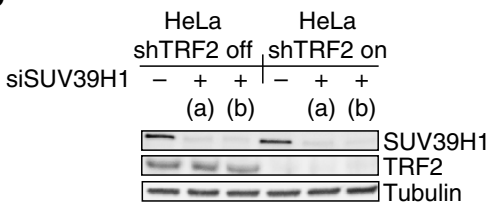

d

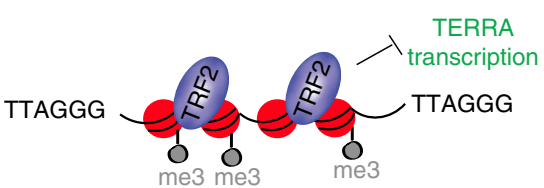

M TERRA transcription

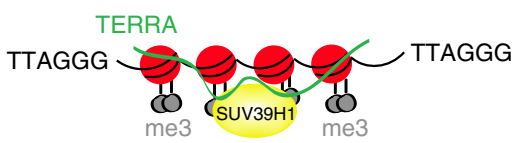

$\uparrow \uparrow$ Telomere fusions

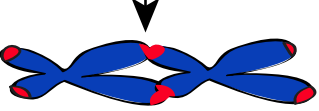

Figure 8 | SUV39H1 sustains telomere end fusions occurring upon TRF2 depletion. (a) Timeline of the experiment in b and c). (b) Western blot of total protein extracts from HeLa cells with or without shTRF2 expression and siRNAs transfection. Uncropped blots are shown in Supplementary Fig. 9d. (c) Metaphase chromosome spreads and telomere-FISH performed on HeLa cells depleted (shTRF2 on) or not depleted (shTRF2 off) for TRF2 and concomitantly transfected with siRNAs against luciferase (siLuc) or SUV39H1 (siSUV39H1a and siSUV39H1b). (d) Model for the regulation and heredescribed roles of TERRA at dysfunctional telomeres.

responsible for the methylation of $\mathrm{H} 3 \mathrm{~K} 9$. Given that telomeric chromatin requires dynamic remodelling upon DNA damage, the TERRA RNA binding ability of SUV39H1 will likely serve to enhance SUV39H1 telomere association following TRF2 depletion promoting de novo methylation of H3K9. Depletion of SUV39H1 reduced telomere fusion events at TRF2-depleted telomeres indicating that SUV39H1 and possibly accumulation of $\mathrm{H} 3 \mathrm{~K} 9 \mathrm{me} 3$ are required for efficient end fusions (this study and ref. 46). Whether this mechanism involves also activation of Tip60 as seen at canonical DNA DSBs remains to be determined in future experiments.

In summary, we provide a comprehensive analysis of the molecular mechanisms underlying TERRA transcriptional regulation at uncapped telomeres and we propose a model in which TERRA acts in the initiation steps of the DDR pathways promoting changes in the chromatin architecture of damaged telomeres and ensuring an effective DDR (Fig. 8d).

\section{Methods}

Plasmids. shRNA vectors were prepared by cloning double-stranded DNA oligonucleotides into pSuper-Puro or pSuper-Blast. The target sequences were as follows: TRF2, 5'-GCGCATGACAATAAGCAGA-3' (ref. 50); POT1 $5^{\prime}$-GTACTA GAAGCCTATCTCA- $3^{\prime}$ (shPOT1-2) and $5^{\prime}$-GGGTGGTACAATTGTCAAT- $3^{\prime}$ (shPOT1-3) (ref. 51); MRE11, 5'-TGAGAACTCTTGGTTTAAC-3' (ref. 50); NBS1, $5^{\prime}$-AGGAAGATGTCAATGTTAG-3' (ref. 50); ATM, 5'-GCAACATACTA CTCAAAGA-3' (ref. 52); ATR, 5' - GGAGATTTCCTGAGCATGT-3' (ref. 53) TRF1, 5'-GAATATTTGGTGATCCAAA-3' (ref. 54); hRAP1 5'-AGAGTTCTTGC ATTGGAACT- $3^{\prime}$ (ref. 55); TIN2 $5^{\prime}$-GTGGAACATTTTCCGCGAGTACTGG AGT-3' (ref. 51); $5^{\prime}$-GACUUAGAUGUUCAGAAAA-3' (ref. 51); hSMG1a 5'-ATC TTCTGGGAATATTACT- $3^{\prime}$ (ref. 23); hSMG1b $5^{\prime}$-CACTTCAGATAACTGA GAG-3' (ref. 23). Full-length human TRF1 and TRF2, and chimeric TRF2 constructs were cloned from cDNAs into pCDNA6-based mammalian expression vectors using PCR amplification and InFusion cloning (Clonetech). The full-length SUV39H1 and SUV39H1 deletion mutant cDNA fragments were cloned into bacterial expression plasmids pGEX6p-1.

Cell culture and transfection. HeLa cells were transfected using Lipofectamine 2000 according to the manufacturer's protocol (Invitrogen). Puromycin

$\left(2 \mu \mathrm{g} \mathrm{ml}^{-1}\right.$; InvivoGen) or blasticidin $\left(10 \mu \mathrm{g} \mathrm{ml}^{-1}\right.$; InvivoGen) was added to the medium $24 \mathrm{~h}$ after transfection of pSuper-Puro and/or pSuper-Blast constructs. Puromycin and blasticidin selection was maintained for 4 days.

siRNA transfections. siRNA transfection was performed with Lipofectamine RNAiMAX (Invitrogen, USA) according to the manufacturer's procedures. SiRNA oligonucleotides sequences were as follows: SUV39H1 siRNAa, 5'-ACCUCUUU GACCUGGACUA-3' (ref. 56), SUV39H1 siRNAb, 5'-CAAAUCGUGUGGUA CAGAA-3' (ref. 57), Luciferase siRNA, 5'-CGUACGCGGAAUACUUCGA-3'.

Affinity purification of UUAGGG-repeat containing RNA. Affinity purification of $5^{\prime}$ capped RNAs was performed by incubating nuclear RNA with GST-eIF4E as described ${ }^{28}$. Briefly, purified GST-eIF4E $(1 \mathrm{mg})$ was bound to glutathione agarose beads in $1 \times$ phosphate-buffered saline (PBS) for $1 \mathrm{~h}$ at $4{ }^{\circ} \mathrm{C}$. The beads were washed with $1 \times$ PBS and with buffer A (10 mM KHPO4 pH 8.0, $100 \mathrm{mM} \mathrm{KCl}$, $2 \mathrm{mM}$ EDTA, $5 \%$ glycerol, $100 \mu \mathrm{g} \mathrm{ml}^{-1}$ yeast tRNA, $6 \mathrm{mM}$ DTT, $1.3 \%$ polyvinyl alcohol, Triton $\mathrm{X}-100,1 \mathrm{U}^{-1} \mathrm{l}^{-1}$ SuperaseIN (Ambion)) and then incubated with nuclear RNA, pre-heated to $75^{\circ} \mathrm{C}$ for $5 \mathrm{~min}$, at room temperature. After $1 \mathrm{~h}$, the supernatant was collected and the beads were washed five times with $1 \mathrm{ml}$ buffer A lacking yeast tRNA. RNA retained on the beads as well as RNA present in the supernatant fraction was extracted using the RNeasy Mini Kit (Qiagen), according to the manufacturer instructions. A total of $125 \mu \mathrm{g}$ of nuclear RNA pulled down with GST-eIF4E was then incubated with 125 pmol of (CCCTAA) $)_{3}$ oligonucleotide

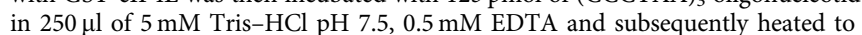
$75^{\circ} \mathrm{C}$ for $5 \mathrm{~min}$ to denature the RNA secondary structures. $\mathrm{NaCl}$ was added to a final concentration of $1 \mathrm{M}$ and the sample was cooled down to $50^{\circ} \mathrm{C}$ over a period of $30 \mathrm{~min}$. Then, $25 \mu \mathrm{l}$ of Dynabeads $\left(10 \mathrm{mg} \mathrm{ml}^{-1}\right.$; Invitrogen) were added and the mixture was incubated at $50{ }^{\circ} \mathrm{C}$ for $3 \mathrm{~h}$. After washing the beads, the RNA was eluted by adding $100 \mu \mathrm{l}$ of $10 \mathrm{mM}$ Tris- $\mathrm{HCl} \mathrm{pH} 8.0$ and heating to $80^{\circ} \mathrm{C}$ for $3 \mathrm{~min}$. 
DSN library preparation and RNA high-throughput sequencing. Libraries were prepared starting from $100 \mathrm{ng}$ of RNA using the PE-102-1002-Paired-end kit from Illumina (Illumina, San Diego, CA, USA) according to manufacturer's recommendations. Ligation products of around $250 \mathrm{bp}$ were gel purified before PCR amplification (15 cycles). Then $100 \mathrm{ng}$ of libraries were normalized according to the Illumina DSN protocol using the Duplex-specific nuclease from Evrogen (JSC, Moscow, Russia). Twelve PCR cycles were performed using PCR PE 1.0 and 2.0 primers to enrich DNA fragments. DSN libraries were loaded on a HiSeq 2000 (Illumina) at $6 \mathrm{pM}$ and paired-end sequenced using the $100 \mathrm{bp}$ protocol. The RNA-seq data sets were submitted to NCBI GEO and have the accession number GSE56727.

Mapping RNA-seq to human subtelomeres. The sequencing produced 100 nucleotide (nt) paired-end reads from each of the four different samples. FLASH $^{58}$ was used to merge overlapping mates into single-end reads. We used FastQC (http://www.bioinformatics.babraham.ac.uk/projects/fastqc) for the quality control. After sequencing quality control, the first 10 and the last five bases were trimmed, leaving read lengths of 85 nucleotides. Except for telomere 1p, the reads were subsequently mapped to a set of 500,000 nt-sized subtelomere assemblies (Wistar Institute, http://www.wistar.org/lab/harold-c-riethman-phd/page/subtelomereassemblies) with Bowtie ${ }^{59}$ (via the HTSstation ${ }^{60}$ ). Coordinate 1 of each completed assembly corresponds to the start of the terminal repeat tract on the strand oriented towards the centromere (to maintain a consistent starting coordinate for subtelomere annotation). For the telomeres whose reference sequence does not extend to the terminal repeat $(6 \mathrm{p}, 8 \mathrm{p}, 11 \mathrm{p}, 3 \mathrm{q}, 9 \mathrm{q}, 20 \mathrm{p})$, coordinate 1 corresponds to the most distal base of the subtelomere assembly. The five acrocentric short-arm telomeres are not represented in these assemblies; while they are known to contain a characteristic SRE organization closely related to distal $4 \mathrm{p}$ (ref. 61), they cannot be distinguished from each other and assemblies adjacent to them are unavailable. For telomere $1 \mathrm{p}$, the reads were mapped to the subtelomere assembly of NCBI (hg19).

Read density profiles were produced counting the number of reads mapping at each position, each read accounting for $1 / N$, where $N$ is the number of its different mapping positions. Scores were further normalized by dividing them by the total number of aligned reads for the sample. The ratio plots represent, at each base position within $50 \mathrm{~kb}$ from the telomere, the value of one of the ratios in log scale. In cases where the denominator was null, the accounted score was arbitrarily chosen as the log of the numerator. RPKMs of housekeeping genes were calculated using portions of the genome covered by the exons of a given gene (not the whole regions between the Ensembl gene coordinates) as described ${ }^{33}$, whereas RPKM values for TERRA molecules were calculated using the length of each subtelomeric region transcribed into different TERRA species.

Northern blot and Real-time RT-PCR. Cells were trypsinized and RNA was prepared using the RNeasy Mini Kit (Qiagen), according to the manufacturer's instructions. For northern blots, RNA $(\sim 5 \mu \mathrm{g})$ was loaded onto $1.3 \%$ formaldehyde agarose gels and separated by electrophoresis. RNA was then transferred to Nylon membranes and blots were blocked in Church buffer for $1 \mathrm{~h}$ at $55^{\circ} \mathrm{C}$ and then incubated with ${ }^{32} \mathrm{P}$-labelled probes. For RT-PCR analysis, total RNA was reverse transcribed with beta-actin ${ }^{28}$ and telomere-specific primers ((CCCTAA)5) at $55^{\circ} \mathrm{C}$ using SuperScript III reverse transcriptase (Invitrogen). For SYBR green reactions, four different TERRA transcripts were analysed as described previously ${ }^{28}$.

Chromatin immunoprecipitation. ChIP assays were performed as described previously $y^{51}$. For immunoprecipitations, $5 \mu \mathrm{g}$ of each specific antibody was used, and the mixtures were incubated overnight at $4{ }^{\circ} \mathrm{C}$ with $50 \mu$ l of a $50 \%$ slurry of protein G-Sepharose beads (GE Healthcare). Telomeric DNA was detected with a probe generated as follows: a template mixture of $1-5 \mathrm{~kb}$ long telomeric DNA fragments was synthesized by ligating double-stranded telomeric DNA oligonucleotides ((TTAGGG)5, (CCCTAA)5) that were amplified by PCR. The probe was random labelled with $\alpha^{-32} \mathrm{P}$-dCTP and cold dTTP and dATP (for detection of the TTAGGG strand). For detection of centromeric sequences, a $5^{\prime}$ ${ }^{32} \mathrm{P}$-labelled oligonucleotide probe corresponding to the alphoid element $\left(5^{\prime}\right.$-GTT TTGAAACACTCTTTTTGTAGAATCTGC-3') was used.

RNA-IP. RNA-IP of formaldehyde-crosslinked extract was performed as described previously ${ }^{29}$. Briefly, for immunoprecipitations, $5 \mu \mathrm{g}$ of SUV39H1 or H3K9me3 antibodies were used, and the mixtures were incubated overnight at $4{ }^{\circ} \mathrm{C}$ with $50 \mu \mathrm{l}$ of a $50 \%$ slurry of protein G-Sepharose beads (GE Healthcare). TERRA was detected with the telomeric probe as described above. For detection of $18 \mathrm{~S}$ rRNA, a specific antisense ${ }^{32} \mathrm{P}-\gamma \mathrm{ATP} 5^{\prime}$-end-labelled oligonucleotide probe was used.

Electromobility shift assay. EMSA with GST-SUV39H1 was performed in $1 \times$ EMSA reaction buffer $(25 \mathrm{mM}$ Tris- $\mathrm{HCl} \mathrm{pH} 8.0,100 \mathrm{mM} \mathrm{NaCl}, 10 \%$ glycerol, $2 \mathrm{mM} \mathrm{MgCl} 2,1 \mathrm{mM}$ DTT, $1 \mathrm{U} \mu \mathrm{l}^{-1}$ SuperaseIN). One nanomolar of ${ }^{32} \mathrm{P}-\gamma \mathrm{ATP}$ $5^{\prime}$-end-labelled RNA or DNA oligonucleotide probes was mixed with the indicated proteins and competitor oligonucleotides and incubated to equilibrium for $30 \mathrm{~min}$ at $37^{\circ} \mathrm{C}$. The reaction was supplemented with EMSA loading buffer to a final

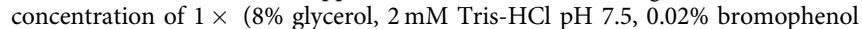
blue and cyan cyanol) and separated on $2 \% 0.5 \times$ TBE-agarose gels at $60 \mathrm{~mA}$ for $30 \mathrm{~min}$. Gels were dried, exposed to Phosphoimager screens and analysed using a FLA-3000 Phosphoimager (Fujifilm) and AIDA Image Analyzer software (Raytest).

Antibodies. The following antibodies were used: Histone H3 (ab1791, Abcam); H3K4mel (07-436, Millipore); H3K4me2 (07-030, Millipore); H3K4me3 (ab8580, Abcam); H3K9me1 (ab9045, Abcam); H3K9me2 (ab1220, Abcam); H3K9me3 (ab8898, Abcam); SUV39H1\#1 (07-958, Millipore); SUV39H1\#2 (Active Motif); MRE11 (NB100-142, Novus Biologicals); NBS1 (NB100-143, Novus Biologicals); ATM (Ab-3, Calbiochem); ATR (N19, SantaCruz); phospho- $\gamma$ H2AX (05-636, Millipore), UPF1 (generous gift of Dr. Claus Azzalin) ${ }^{62} ; \alpha-$ phospho-(Ser/Thr) ATM/ATR substrate (6966, Cell Signaling).

Microscopy. IF-FISH staining and telomere-FISH analysis of chromosome metaphase spreads were performed as described ${ }^{63}$. Briefly, for IF-FISH, cover slips were washed with $1 \mathrm{ml} 1 \times \mathrm{PBS}$ and then fixed with $4 \%$ formaldehyde in $1 \times \mathrm{PBS}$ for $10 \mathrm{~min}$ at room temperature. Subsequently, the cover slips were washed with $1 \times$ PBS, permeabilized in $1 \times$ detergent solution $(0.1 \%$ Triton $\mathrm{X}-100,0.02 \%$ SDS in $1 \times$ PBS) and then blocked with $10 \%$ normal serum in BSA $/ 1 \times$ PBS. Subsequently, cover slips were incubated with primary and then with secondary antibody, fixed with $4 \%$ formaldehyde in $1 \times$ PBS and dehydrated with ethanol. For FISH staining, cover slips were incubated with the hybridization mix,

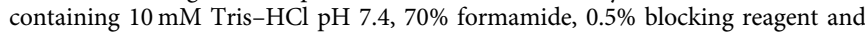
$1 / 1,000 \mathrm{Cy} 3$ probe, denatured at $80^{\circ} \mathrm{C}$ and hybridized for $3 \mathrm{~h}$ at room temperature. After hybridization, cover slips were washed, stained with DAPI and dehydrated with ethanol. For metaphase telomere-FISH, HeLa cells were treated with $0.05 \mu \mathrm{g}$ Demecolcine for $2 \mathrm{~h}$. Subsequently cells were collected, resuspended in $56 \mathrm{mM} \mathrm{KCl}$ at $37^{\circ} \mathrm{C}$ for $5 \mathrm{~min}$ and then fixed with methanol/acid acetic 3:1 solution. Resuspended cells were dropped onto wet slides, fixed with $4 \%$ formaldehyde in $1 \times$ PBS and dehydrated with ethanol. The FISH procedure was performed as described above.

\section{References}

1. Cann, K. L. \& Dellaire, G. Heterochromatin and the DNA damage response: the need to relax. Biochem. Cell Biol. 89, 45-60 (2011).

2. Ward, G. K., Stewart, S. S., Price, G. B. \& Mackillop, W. J. Cellular heterogeneity in normal human urothelium: quantitative studies of lectin binding. Histochem. J. 19, 337-344 (1987).

3. Shanbhag, N. M., Rafalska-Metcalf, I. U., Balane-Bolivar, C., Janicki, S. M. \& Greenberg, R. A. ATM-dependent chromatin changes silence transcription in cis to DNA double-strand breaks. Cell 141, 970-981 (2010).

4. Price, B. D. \& D'Andrea, A. D. Chromatin remodeling at DNA double-strand breaks. Cell 152, 1344-1354 (2013).

5. Greenberg, R. A. Histone tails: directing the chromatin response to DNA damage. FEBS Lett. 585, 2883-2890 (2011).

6. Goodarzi, A. A. et al. ATM signaling facilitates repair of DNA double-strand breaks associated with heterochromatin. Mol. Cell 31, 167-177 (2008).

7. Ayoub, N., Jeyasekharan, A. D., Bernal, J. A. \& Venkitaraman, A. R. HP1-beta mobilization promotes chromatin changes that initiate the DNA damage response. Nature 453, 682-686 (2008).

8. Murr, R. et al. Histone acetylation by Trrap-Tip60 modulates loading of repair proteins and repair of DNA double-strand breaks. Nat. Cell Biol. 8, 91-99 (2006).

9. Bird, A. W. et al. Acetylation of histone $\mathrm{H} 4$ by Esal is required for DNA double-strand break repair. Nature 419, 411-415 (2002).

10. Sun, Y. et al. Histone H3 methylation links DNA damage detection to activation of the tumour suppressor Tip60. Nat. Cell Biol. 11, 1376-1382 (2009).

11. Sun, Y., Xu, Y., Roy, K. \& Price, B. D. DNA damage-induced acetylation of lysine 3016 of ATM activates ATM kinase activity. Mol. Cell. Biol. 27, 8502-8509 (2007).

12. Kaidi, A. \& Jackson, S. P. KAT5 tyrosine phosphorylation couples chromatin sensing to ATM signalling. Nature 498, 70-74 (2013).

13. Benetti, R. et al. Suv4-20h deficiency results in telomere elongation and derepression of telomere recombination. J. Cell Biol. 178, 925-936 (2007).

14. Hewitt, G. et al. Telomeres are favoured targets of a persistent DNA damage response in ageing and stress-induced senescence. Nat. Commun. 3, 708 (2012),

15. Fumagalli, M. et al. Telomeric DNA damage is irreparable and causes persistent DNA-damage-response activation. Nat. Cell Biol. 14, 355-365 (2012).

16. de Lange, T. Shelterin: the protein complex that shapes and safeguards human telomeres. Genes Dev. 19, 2100-2110 (2005).

17. de Lange, T. How telomeres solve the end-protection problem. Science 326, 948-952 (2009).

18. Denchi, E. L. \& de Lange, T. Protection of telomeres through independent control of ATM and ATR by TRF2 and POT1. Nature 448, 1068-1071 (2007). 
19. Sharpless, N. E. \& DePinho, R. A. Cancer: crime and punishment. Nature 436, 636-637 (2005).

20. Feldser, D. M. \& Greider, C. W. Short telomeres limit tumor progression in vivo by inducing senescence. Cancer Cell 11, 461-469 (2007).

21. Cosme-Blanco, W. et al. Telomere dysfunction suppresses spontaneous tumorigenesis in vivo by initiating p53-dependent cellular senescence. $E M B O$ Rep. 8, 497-503 (2007).

22. d'Adda di Fagagna, F. et al. A DNA damage checkpoint response in telomereinitiated senescence. Nature 426, 194-198 (2003).

23. Azzalin, C. M., Reichenbach, P., Khoriauli, L., Giulotto, E. \& Lingner, J. Telomeric repeat containing RNA and RNA surveillance factors at mammalian chromosome ends. Science 318, 798-801 (2007).

24. Schoeftner, S. \& Blasco, M. A. Developmentally regulated transcription of mammalian telomeres by DNA-dependent RNA polymerase II. Nat. Cell Biol. 10, 228-236 (2008)

25. Nergadze, S. G. et al. CpG-island promoters drive transcription of human telomeres. RNA 15, 2186-2194 (2009).

26. Yehezkel, S., Segev, Y., Viegas-Pequignot, E., Skorecki, K. \& Selig, S. Hypomethylation of subtelomeric regions in ICF syndrome is associated with abnormally short telomeres and enhanced transcription from telomeric regions. Hum. Mol. Genet. 17, 2776-2789 (2008).

27. Arnoult, N., Van Beneden, A. \& Decottignies, A. Telomere length regulates TERRA levels through increased trimethylation of telomeric $\mathrm{H} 3 \mathrm{~K} 9$ and HP1alpha. Nat. Struct. Mol. Biol. 19, 948-956 (2012).

28. Porro, A., Feuerhahn, S., Reichenbach, P. \& Lingner, J. Molecular dissection of telomeric repeat-containing RNA biogenesis unveils the presence of distinct and multiple regulatory pathways. Mol. Cell. Biol. 30, 4808-4817 (2010).

29. Deng, Z., Norseen, J., Wiedmer, A., Riethman, H. \& Lieberman, P. M. TERRA RNA binding to TRF2 facilitates heterochromatin formation and ORC recruitment at telomeres. Mol. Cell 35, 403-413 (2009).

30. Hayashi, M. T., Cesare, A. J., Fitzpatrick, J. A., Lazzerini-Denchi, E. \& Karlseder, J. A telomere-dependent DNA damage checkpoint induced by prolonged mitotic arrest. Nat. Struct. Mol. Biol. 19, 387-394 (2012).

31. Stong, N. et al. Subtelomeric CTCF and cohesin binding site organization using improved subtelomere assemblies and a novel annotation pipeline. Genome Res. 24, 1039-1050 (2014).

32. Deng, Z. et al. A role for CTCF and cohesin in subtelomere chromatin organization, TERRA transcription, and telomere end protection. EMBO J. 31, 4165-4178 (2012)

33. Mortazavi, A., Williams, B. A., McCue, K., Schaeffer, L. \& Wold, B. Mapping and quantifying mammalian transcriptomes by RNA-Seq. Nat. Methods 5, 621-628 (2008)

34. Hann, S. R. \& Eisenman, R. N. Proteins encoded by the human c-myc oncogene: differential expression in neoplastic cells. Mol. Cell. Biol. 4, 2486-2497 (1984).

35. King, M. W., Roberts, J. M. \& Eisenman, R. N. Expression of the c-myc proto-oncogene during development of Xenopus laevis. Mol. Cell. Biol. 6, 4499-4508 (1986).

36. Waters, C. M., Littlewood, T. D., Hancock, D. C., Moore, J. P. \& Evan, G. I. c-myc protein expression in untransformed fibroblasts. Oncogene 6, 797-805 (1991).

37. Yamashita, A., Ohnishi, T., Kashima, I., Taya, Y. \& Ohno, S. Human SMG-1, a novel phosphatidylinositol 3-kinase-related protein kinase, associates with components of the mRNA surveillance complex and is involved in the regulation of nonsense-mediated mRNA decay. Genes Dev. 15, 2215-2228 (2001).

38. Brumbaugh, K. M. et al. The mRNA surveillance protein hSMG-1 functions in genotoxic stress response pathways in mammalian cells. Mol. Cell 14, 585-598 (2004).

39. Bunz, F. et al. Requirement for p53 and p21 to sustain G2 arrest after DNA damage. Science 282, 1497-1501 (1998).

40. Fairall, L., Chapman, L., Moss, H., de Lange, T. \& Rhodes, D. Structure of the TRFH dimerization domain of the human telomeric proteins TRF1 and TRF2. Mol. Cell 8, 351-361 (2001).

41. Baker, A. M. et al. The telomere binding protein TRF2 induces chromatin compaction. PLoS ONE 6, e19124 (2011).

42. Poulet, A. et al. The N-terminal domains of TRF1 and TRF2 regulate their ability to condense telomeric DNA. Nucleic Acids Res. 40, 2566-2576 (2012)

43. Broccoli, D., Smogorzewska, A., Chong, L. \& de Lange, T. Human telomeres contain two distinct Myb-related proteins, TRF1 and TRF2. Nat. Genet. 17, 231-235 (1997)

44. Okamoto, K. et al. A two-step mechanism for TRF2-mediated chromosomeend protection. Nature 494, 502-505 (2013).

45. Grolimund, L. et al. A quantitative telomeric chromatin isolation protocol identifies different telomeric states. Nat. Commun. 4, 2848 (2013).

46. Bartocci, C. et al. Isolation of chromatin from dysfunctional telomeres reveals an important role for Ringlb in NHEJ-mediated chromosome fusions. Cell Rep. 7, 1320-1332 (2014).
47. Caslini, C., Connelly, J. A., Serna, A., Broccoli, D. \& Hess, J. L. MLL associates with telomeres and regulates telomeric repeat-containing RNA transcription. Mol. Cell. Biol. 29, 4519-4526 (2009).

48. de Lange, T. T-loops and the origin of telomeres. Nat. Rev. Mol. Cell Biol. 5, 323-329 (2004).

49. Doksani, Y., Wu, J. Y., de Lange, T. \& Zhuang, X. Super-resolution fluorescence imaging of telomeres reveals TRF2-dependent T-loop formation. Cell 155, 345-356 (2013).

50. Porro, A., Feuerhahn, S. \& Lingner, J. TERRA-reinforced association of LSD1 with MRE11 promotes processing of uncapped telomeres. Cell Rep. 6, 765-776 (2014).

51. Abreu, E. et al. TIN2-tethered TPP1 recruits human telomerase to telomeres in vivo. Mol. Cell. Biol. 30, 2971-2982 (2010).

52. Xu, L. \& Blackburn, E. H. Human cancer cells harbor T-stumps, a distinct class of extremely short telomeres. Mol. Cell 28, 315-327 (2007).

53. Azzalin, C. M. \& Lingner, J. The human RNA surveillance factor UPF1 is required for S phase progression and genome stability. Curr. Biol. 16, 433-439 (2006).

54. McKerlie, M. \& Zhu, X. D. Cyclin B-dependent kinase 1 regulates human TRF1 to modulate the resolution of sister telomeres. Nat. Commun. 2, 371 (2011).

55. Crabbe, L., Cesare, A. J., Kasuboski, J. M., Fitzpatrick, J. A. \& Karlseder, J. Human telomeres are tethered to the nuclear envelope during postmitotic nuclear assembly. Cell Rep. 2, 1521-1529 (2012).

56. Liu, B. et al. Depleting the methyltransferase Suv39h1 improves DNA repair and extends lifespan in a progeria mouse model. Nat. Commun. 4, 1868 (2013)

57. Ayrapetov, M. K., Gursoy-Yuzugullu, O., Xu, C., Xu, Y. \& Price, B. D. DNA double-strand breaks promote methylation of histone $\mathrm{H} 3$ on lysine 9 and transient formation of repressive chromatin. Proc. Natl Acad. Sci. USA 111, 9169-9174 (2014).

58. Magoc, T. \& Salzberg, S. L. FLASH: fast length adjustment of short reads to improve genome assemblies. Bioinformatics 27, 2957-2963 (2011).

59. Langmead, B., Trapnell, C., Pop, M. \& Salzberg, S. L. Ultrafast and memoryefficient alignment of short DNA sequences to the human genome. Genome Biol. 10, R25 (2009).

60. David, F. P. et al. HTSstation: a web application and open-access libraries for high-throughput sequencing data analysis. PLoS ONE 9, e85879 (2014).

61. Youngman, S. et al. The telomeric $60 \mathrm{~kb}$ of chromosome arm $4 \mathrm{p}$ is homologous to telomeric regions on 13p, 15p, 21p, and 22p. Genomics 14, 350-356 (1992)

62. Chawla, R. et al. Human UPF1 interacts with TPP1 and telomerase and sustains telomere leading-strand replication. EMBO J. 30, 4047-4058 (2011).

63. Celli, G. B. \& de Lange, T. DNA processing is not required for ATM-mediated telomere damage response after TRF2 deletion. Nat. Cell Biol. 7, 712-718 (2005).

\section{Acknowledgements}

We thank S. Redon and P. Reichenbach for initial observations indicating a role of TRF2 in TERRA repression; M. Docquier, P. Descombes and C. Delucinge-Vivier from the iGE3 genomics platform (University of Geneva) for performing RNA-seq; I. Zemp for technical help; E. Aeby for the inducible TRF2-KD HeLa cells; C. M. Azzalin for antiUPF1 antibodies; B. Vogelstein for HCT116 and HCT116 p53 KO cells; L. Chen, L. Grolimund and C.M. Azzalin for sharing reagents and discussions. A.P. was, in part, supported by an EMBO postdoctoral fellowship; and S.F. was, in part, supported by a postdoctoral fellowship from the DFG (FE 1205/1-1). H.R. was supported by NIH HG007205. Research in J.L.'s laboratory was supported by a grant from the Swiss National Science Foundation, the NCCR RNA and Disease funded by the Swiss National Science Foundation, a European Research Council advanced investigator grant (grant agreement number 232812), an Initial Training Network (ITN) grant (CodeAge) from the European Commission's Seventh Framework Programme (grant agreement number 316354), the Swiss Cancer League and EPFL.

\section{Author contributions}

A.P. and S.F. performed the experiments and analysed the data. J.D., J.R. and H.R performed the RNA-seq analysis. A.P. and J.L. designed the study and wrote the paper.

\section{Additional information}

Accession codes: The TERRA sequence data have been deposited in NCBI's Gene Expression Omnibus and are accessible through GEO Series accession number GSE56727 (http://www.ncbi.nlm.nih.gov/geo/query/acc.cgi?acc = GSE56727).

Supplementary Information accompanies this paper at http://www.nature.com/ naturecommunications

Competing financial interests: The authors declare no competing financial interests.

Reprints and permission information is available online at http://npg.nature.com/ reprintsandpermissions/

How to cite this article: Porro, A. et al. Functional characterization of the TERRA transcriptome at damaged telomeres. Nat. Commun. 5:5379 doi: 10.1038/ncomms6379 (2014). 